\title{
Proliferation and Survival of T-cell Acute Lymphoblastic Leukaemia Depends on mTOR-regulated Glutamine Uptake and EAAT1-dependent Conversion of Glutamine to Aspartate and Nucleotides
}

Vesna S. Stanulović ${ }^{1}$, Michelle A.C. Reed ${ }^{1}$, Hemalvi Patani ${ }^{1,5}$, Sandeep Potluri ${ }^{1}$, Eleni Georgiadou ${ }^{1,6}$, Jennie Roberts ${ }^{1}$, Sovan Sarkar ${ }^{1}$, Guy Pratt ${ }^{1,2}$, Alan M. Jones ${ }^{3}$, Ulrich Günther ${ }^{1}$, Christian Ludwig ${ }^{4}$ and Maarten Hoogenkamp ${ }^{1, *}$

${ }^{1}$ Institute of Cancer and Genomic Sciences, University of Birmingham, Birmingham, West Midlands, B15 2TT, United Kingdom

${ }^{2}$ Centre for Clinical Haematology, Queen Elizabeth Hospital Birmingham, West Midlands, B15 2GW, Birmingham, United Kingdom

${ }^{3}$ School of Pharmacy, University of Birmingham, Birmingham, West Midlands, B15 2TT, United Kingdom

${ }^{4}$ Institute of Metabolism and Systems Research, University of Birmingham, Birmingham, West Midlands, B15 2TT, United Kingdom

${ }^{5}$ Current address: Bart's Cancer Institute, Queen Mary University of London, London, Greater London, EC1M 6BQ, United Kingdom

${ }^{6}$ Current address: Division of Diabetes, Endocrinology and Metabolism, Department of Medicine, Imperial College London, London, Greater London, W12 0NN, United Kingdom.

*Correspondence: $\underline{\text { m.hoogenkamp@bham.ac.uk }}$ 


\section{Summary}

T-cell acute lymphoblastic leukaemia (T-ALL) is a cancer of the immune system. Approximately $20 \%$ of paediatric and $50 \%$ of adult T-ALL patients relapse and die from the disease. To improve patient outcome new drugs are needed. With the aim to identify new therapeutic targets, we integrated transcriptomics and metabolomics data, including live-cell NMR-spectroscopy, of cell lines and patient samples. We found that T-ALL cells have limited energy availability, resulting in active AMPK-signalling and reduced autophagy. Despite this, mTOR kinase remains active and essential for glutamine-uptake that fuels rapid proliferation. Glutamine fuels aspartate synthesis and together they supply three nitrogen atoms in purines and all atoms but one carbon in pyrimidine rings. We show that EAAT1, a glutamateaspartate transporter normally only expressed in the CNS, is crucial for glutamine conversion to nucleotides and that T-ALL cell proliferation depends on EAAT1 function, identifying it as a target for T-ALL treatment. Finally, we performed an in silico screen and identified a novel EAAT1-specific allosteric inhibitor.

Key words: T-ALL, T-cell Acute Lymphoblastic Leukaemia, mTOR, AMPK, lysosome, NMR spectroscopy, glutamine, glutamate, aspartate, live-cell NMR, de novo nucleotide synthesis, SLC1A3, EAAT1, EAA1, GLAST-1 


\section{Introduction}

For a cancer to proliferate, cells need to increase their size and replicate their DNA, which requires large quantities of proteins, lipids and nucleotides, as well as energy. Metabolic changes and adaptations during tumorigenesis enable tumour cell growth and survival by altering metabolic pathways, such as glycolysis and glutaminolysis [1-3], and signal transduction cascades that regulate metabolic processes, such as the PI3K-AKT-mTOR pathway [4-6]. The compilation of the metabolic changes results in a distinct metabolic phenotype. Annotation of the rate-limiting steps that support the oncogenic metabolic adaptations can lead to the identification of useful therapeutic targets for the development of new anti-cancer therapies.

In order to understand and identify oncogenic processes that support rapid proliferation, we developed a methodology that assessed and integrated malignant gene expression, signal transduction and metabolism. We used T-cell acute lymphoblastic leukaemia (T-ALL) as a model. T-ALL is a haematological malignancy of the T-cell lineage that occurs in adults and children. Gene expression profiling studies showed that paediatric T-ALL patients can be grouped into four major clusters: TAL/LMO, TLX/HOXA, Proliferative and Immature (ETP ALL) $[7,8]$. Most T-ALL have additional mutations in genes instrumental for signal transduction pathways such as NOTCH (>60\%), PTEN-PI3K-AKT, JAK-STAT and FBXW7 $[9,10]$. Monitoring of minimal residual disease (MRD) is a response based treatment protocol that is used for T-ALL patient stratification into standard, intermediate and high risk groups [11]. Even though treatment outcome has improved, approximately $20 \%$ of paediatric and $50 \%$ of adult T-ALL patients have refractory disease or relapse and most of these die from the disease $[12,13]$. Refractory disease is more frequent in T-ALL displaying an early T-cell phenotype, which most commonly occurs in ETP and TAL/LMO T-ALL [14, 15]. SIL/TAL chromosomal microdeletion (1p32q) is the most common genetic rearrangement in T-ALL [7]. SIL-TAL deletion positions the promoter of the constitutively active STIL gene in front of the coding sequences of the TAL1 transcription factor that is crucial for haematopoietic and T-cell development and differentiation [16].

In this study we integrated gene expression data, signal transduction activity and metabolic studies, using cell lines and purified primary patient samples. We found that T-ALL cell cycle progression depends on mTOR-dependent glutamine uptake. Employing metabolic labelling we found that glutamine provides all nitrogen atoms and all but one carbon in the pyrimidine ring and three of the four nitrogens in purines. Furthermore, analyses of the metabolic processes supporting glutamine metabolic pathways and the gene expression of T-ALL cell lines and patient samples showed that T-ALL cells express the glutamate-aspartate antiporter EAAT1 (encoded by SLC1A3) [8]. In the healthy adult body, EAAT1 is only 
present in the neurons and glia of the central nervous system where it is required for the uptake of the cytotoxic glutamate from the glutamatergic synapses in exchange for aspartate $[17,18]$. In T-ALL, EAAT1 is required for glutamate import into mitochondria in exchange for glutamine-derived aspartate that is used as a substrate for nucleotide production in the cytoplasm. We show that T-ALL survival depends on EAAT1 function which validates EAAT1 as a therapeutic target for treating T-ALL. Subsequently, we performed a focussed small molecule screen and identified EAAT1inh_1 as a novel and potent EAAT1-specific allosteric inhibitor.

\section{Results}

\section{SIL-TAL cells exhibit suppressed carbohydrate and upregulated nucleotide metabolism}

Based on the prevalence and the risk of refraction/relapse, we focussed our efforts on ETP and SIL-TAL T-ALL. We chose three cell lines that carry the SIL-TAL rearrangement (DU.528, HSB2 and CCRF-CEM) and one cell line without, which immuno-phenotypically belongs to the ETP T-ALL group (ARR) [19-23]. RNA-seq identified 4508 differentially expressed genes between the cell lines. Principal component analysis (PCA) and hierarchical clustering found that the three SIL-TAL cell lines clustered separately from ARR, with HSB2 and CCRF_CEM being most similar (Figure 1A,S1) Hierarchical clustering grouped differentially expressed genes into 11 groups (Figure 1A). Genes suppressed in the SIL-TAL cells grouped in cluster 1 (C1), whereas $\mathrm{C} 9$ and $\mathrm{C} 10$ comprised upregulated genes. Gene functional annotation analyses indicated that ARR and SIL-TAL cells had a number of differentially regulated metabolic pathways (Figure 1B,S2). C1 was associated with carbohydrate catabolism, protein processing in the endoplasmic reticulum and amino-acid biosynthesis, whereas $\mathrm{C} 10$ involved purine and pyrimidine synthesis, sphingolipid metabolism and C9 contained T cell receptor and Rap1 signalling (Figure 1B). Additionally, genes that were upregulated in at least two of the SIL-TAL cell lines (C8 and C11) were implicated in signal transduction pathways such as MAPK, Hippo, Rap-1 (C8) and TGF- $\beta$, Ras, Rap-1, JAK-STAT, AMPK, mTOR (C11) (Figure S2). Based on this we conclude that the SIL-TAL metabolic phenotype in comparison to ARR is characterised by suppressed utilisation of carbohydrates and amino acid/protein synthesis, and upregulated nucleotide metabolism.

Validation of the RNA-seq by qPCR confirmed differential gene expression for argininosuccinate synthase 1 (ASS1; cluster $\mathrm{C} 1$ ) and Acyl-CoA synthetase short-chain family member 1 (ACSS1; cluster C10), two genes whose products have metabolic functions (Figure 1C,D). ASS1 converts citrulline and aspartate into L-argininosuccinate, an 
intermediate in arginine biosynthesis, which is also important for alanine, aspartate and glutamine metabolism. ACSS1 encodes a protein that catalyses the conversion of acetate to acetyl-CoA [24-26]. ASS1 was only expressed in ARR cells, whereas ACSS1 had the opposite pattern, with higher expression levels found in the SIL-TAL cells (Figure 1C,D).

\section{Protein synthesis is supressed in SIL-TAL cells}

We decided to assess whether the findings of the gene expression analyses were reflected in the T-ALL metabolism. To test if the amino acid synthesis is suppressed in SIL-TAL cell lines, we measured de novo protein synthesis using Click-iT OP-puro methodology [27]. The quantification revealed that SIL-TAL cells exhibited $30-40 \%$ lower protein synthesis than ARR (Figure 2A,B).

The mTOR signalling pathway is the main regulator of protein synthesis based on the availability of amino acids and cellular energy status [28-30]. Based on (i) the downregulated protein synthesis in SIL-TAL, (ii) their inability to produce arginine due to the absence of the ASS1 enzyme and (iii) suppressed amino acid biosynthesis based on the gene ontology, we expected attenuated signalling through the mTOR pathway. To test the importance of the mTOR pathway on T-ALL cells we employed rapamycin, an inhibitor of the mTOR kinase activity. Upon treatment SIL-TAL cell proliferation was reduced, so that after 4 days the number of treated cells was $40 \%$ compared to the vehicle-treated control (Figure 2C) [31, 32]. Rapamycin treatment of ARR did not affect cell proliferation, demonstrating that ARR was insensitive to mTOR inhibition, whereas SIL-TAL cell lines required active mTOR kinase activity for their proliferation.

Lysosomal co-localisation of mTORC1 and its interacting partners on the lysosomal membrane is an additional level of mTOR regulation, which is required for correct mTORC1 signalling and is independent of mTOR kinase activity [33-35]. To examine mTOR localisation under normal growth conditions and upon mTOR inhibition by rapamycin, we imaged localisation of mTOR and the lysosomal marker LAMP1 in parallel with monitoring lysosomal acidification with a live-cell LysoTracker dye (Figure 2D) [36-38]. In all the cell lines mTOR partly co-localised with LAMP1 staining but also had nuclear and cytoplasmic localisation. Surprisingly, upon rapamycin treatment, mTOR was almost exclusively colocalised with punctual LAMP1 staining (Figure 2D). This increase in lysosomal mTOR localisation upon rapamycin treatment reveals that the regulation of mTOR localisation was functional and suggests that the T-ALL cells had limited amino acid availability.

Additionally we observed that SIL-TAL cells had weak to absent LysoTracker staining that was further reduced upon rapamycin treatment, which implies reduced lysosomal acidification (Figure 2D) and could be a possible cause of the limited amino acid availability, 
as acidified lysosomes are required for protein degradation, supplying the cell with free amino acids.

\section{AMPK signalling is active in T-ALL cell lines}

Our results indicate restricted amino acid availability in all T-ALL cell lines and suggest reduced $m$ TOR signalling. In order to gain better understanding of mTOR signalling in the TALL cell lines, we assayed the endogenous levels and phosphorylation status of proteins constituting the PI3K-AKT-mTOR signalling cascade under normal growth conditions and in rapamycin treated cells (Figure S3A,B, Supplementary results). We observed a number of differences between the T-ALL cell lines that show impaired mTOR signalling, including the finding that ARR have constitutively active mTOR due to the lack of TSC1 (Figure S3C,D,E). Common for all the T-ALL cell lines was S792 phosphorylation on Raptor (Figure 2E). Raptor is an mTOR binding partner and regulator which mediates the mTOR interaction with 4E-BP1 and p70S6K [39-42]. As a result of low cellular energy status, AMP kinase (AMPK) phosphorylates S792 on Raptor, leading to mTORC1 inhibition and cell cycle arrest [43]. The observed $\mathrm{p}$-Raptor ${ }^{\mathrm{5} 92}$ implies active AMPK and reduced energy availability in all T-ALL cells.

Both, p-Raptor ${ }^{\mathrm{S} 92}$ and the reduced lysosomal acidification, could be a consequence of active AMPK in T-ALL cells (Figure 2D,E). Indeed, p-AMPK ${ }^{\top 172}$, a mark of AMPK activation, was detected as well as the phosphorylation of AMPK targets, ULK1 ${ }^{\mathrm{S} 555}$ and Beclin-1 ${ }^{\mathrm{S} 93}$ (Figure 2F) [44-48]. ULK1 and Beclin-1 are important for autophagosome formation and initiation of autophagy, a regulated self-digestion that is induced by starvation [49, 50]. Our results demonstrate that all T-ALL cells have activated AMPK and induced autophagy.

\section{Autophagy and lysosomal function is impaired in SIL-TAL}

Even though autophagy is induced in T-ALL, the observed impaired lysosomal acidification in SIL-TAL cells implies that these cells have a limited ability to hydrolyse cargo delivered by autophagosomes, which would cause an obstructed autophagic flux. ULK-1 and Beclin-1 activate the autophagy-related (ATG) protein machinery, resulting in the lipidation of the cytosolic protein LC3-I to LC3-II and integration into autophagosomes (reviewed in [51]). LC3-II interaction with the cargo receptors, such as p62, allows autophagosomes to engulf cytoplasmic components, including cytosolic proteins and organelles. Subsequent fusion with lysosomes facilitates cargo degradation by lysosomal hydrolases and the release of nutrients [51]. In order to determine whether T-ALL cells have effective autophagy, we examined the levels of LC3I, LC3II and the cargo receptor p62, as well as the localisation of LC3II in the control and rapamycin treated cells. All four cell lines exhibited higher LC3I than LC3II levels and punctual cytoplasmic LC3II, which only in ARR overlapped with lysosomal 
LAMP1 staining, revealing the presence of autophagosomes in all T-ALL cells, but fusion with lysosomes only in ARR cells (Figure 3A,B). Rapamycin-induced autophagy in ARR cells lead to elevated production of LC3I and LC3II, hence autophagosomes and their fusion with lysosomes, as seen by increased punctual LC3II staining that co-localised with LAMP1 (Figure 3A,B). ARR also show the lowest levels of p62, confirming effective cargo clearance (Figure 3B). Altogether, these results demonstrate functional autophagy only in ARR. Rapamycin treatment had no noticeable effect on LC3II and p62 abundance in DU.528 and HSB2 cells albeit some increase of LC3Il was observed in CCRF-CEM cells (Figure 3A,B). SIL-TAL cells exhibited punctual LC3II staining, which did not co-localise with LAMP1 and even after rapamycin treatment co-localisation was sporadic. The limited LC3II-LAMP1 colocalisation and reduced lysosomal acidification indicate that SIL-TAL cells have obstructed autophagic clearance.

In order to find out whether reduced lysosomal acidification is the consequence of a low energy status/AMPK activity, or impaired v-ATPase function, we inhibited AMPK using dorsomorphin [52]. Treatment reduced the abundance of LC3I and LC3II in all four T-ALL cell lines while p62 remained constant (Figure 3D). Immuno-fluorescent staining of the treated cells showed that the SIL-TAL cells had functional v-ATPase and the capacity to acidify their lysosomes, as seen by LysoTracker staining accumulated in LAMP1 positive lysosomes, a proportion of which was co-localised with autophagosomal LC3II (Figure 3C). The ARR cell line displayed an increase in the amount of punctual, co-localised LC3II and LAMP1 staining. In line with the western blot analyses these results show that inhibition of AMPK activity leads to lysosomal acidification and activated autophagy in SIL-TAL cells and to a further increase in autophagic activity in ARR cells. Therefore, we conclude that all TALL cell lines have functional v-ATPase and that reduced autophagy is caused by the unfavourable energy status and active AMPK.

\section{mTOR Regulates Glutamine and Glucose Utilisation}

So far, we observed the consequences of limited ATP and amino acid availability on the mTOR/AMPK signalling, autophagy and protein synthesis. Despite this, the T-ALL cell lines undergo rapid cellular proliferation. In order to better understand how the T-ALL cells source metabolites to fuel rapid proliferation, we measured their metabolism by establishing their metabolite uptake/release and their intracellular metabolite levels.

Metabolite uptake was assessed by measuring metabolite levels in the media, $24 \mathrm{~h}$ after medium change, by NMR spectroscopy. We identified 22 metabolites, whose levels were significantly changing in at least one of the T-ALL cell lines. Hierarchical clustering of the observed changes showed that all four cell lines uptake lysine, glucose, phenylalanine and glutamine and release lactate, pyruvate, glutamate and pyroglutamate (Figure 4A). mTOR 
inhibition by rapamycin resulted in reduced glucose and glutamine uptake and lactate release in all T-ALL cells, indicating that glucose and glutamine uptake depended on an active mTOR pathway (Figure 4B).

\section{T-ALL metabolic phenotype}

NMR analysis of intracellular metabolite levels was used to determine differences in metabolite levels between the cell lines, $24 \mathrm{~h}$ after changing the growth medium. Hierarchical clustering of the metabolites that showed significantly different levels between any two cell lines, identified four metabolic clusters MC1-4 (Figure 4C, S4A). MC1 and MC3 were comprised of metabolites more abundant in SIL-TAL cells and ARR, respectively. Phosphatidylcholine, glycerophosphatidylcholine (GPC) and the amino acids alanine, glutamate, aspartate and cysteine-derived taurine were constituents of MC1, while AMP, succinate, $\mathrm{NAD}^{+}$, glutathione, as well as creatine and phosphocreatine were members of MC3. Therefore, direct measurements show that ARR cells have the highest AMP and the lowest ATP/ADP level, confirming that ARR have a low energy status, in line with the active AMPK seen in Figure 2F. Metabolites with low abundance in CCRF-CEM cells were featured in the MC2 cluster. This cluster included glutamine, which similar to MC3 metabolites, was found at the highest level in ARR (Figure 4C). We therefore observe that SIL-TAL have a specific metabolic phenotype, characterised by lower intracellular glutamine levels and higher levels of the glutamine-derived metabolites glutamate, aspartate and alanine. Taking into consideration that (i) T-ALL uptake glutamine, which is a precursor for glutamate and aspartate, (ii) rapamycin reduces glutamine utilisation and (iii) that glutamine, glycine and aspartate are substrates for de novo nucleotide synthesis, glutamine could be the main source for de novo nucleotide synthesis in T-ALL.

Intracellular metabolite levels are very dynamic and change as a response to metabolite uptake and cellular demand. In order to address the dynamics of the metabolite levels, we compared the abundance of intracellular metabolites at the time of growth-media change $(0 \mathrm{~h})$ to that found $8 \mathrm{~h}$ and $24 \mathrm{~h}$ later (Figure $4 \mathrm{D}$ ). A similar response to media change in all four cell lines was found for glutamate and myoinositol, whose availability decreased upon media supplementation. Elevated intracellular concentration of glutamate is consistent with our previous finding that T-ALL release glutamate into the media, as well as glutamatederived pyroglutamate (Figure 4A). A polarised response between SIL-TAL and ARR was observed for AMP, which was the only metabolite with increasing levels in SIL-TAL cells while decreasing in ARR (Figure 4D). Based on the finding that in SIL-TAL cells media change led to increasing AMP levels, while ATP/ADP levels were overall maintained, we conclude that SIL-TAL cells have an increasing AMP to ATP/ADP ratio upon medium 
change. This supports our finding that SIL-TAL cells had active AMPK and reduced energy availability, as described above (Figure $2 \mathrm{~F}$ ).

Opposite dynamics were observed between ARR and SIL-TALs for NAD , tyrosine, isoleucine, leucine, phenylalanine, valine and glutamine, whose concentrations were lower in SIL-TAL at $8 \mathrm{~h}$ and/or $24 \mathrm{~h}$, and higher in ARR. This reduction of the intracellular abundance of essential amino acids is an experimental observation that directly corroborates our previous conclusion that SIL-TAL have reduced amino acid availability (Figure 4D,2A,D). In $A R R$, the levels of these metabolites were elevated at $8 \mathrm{~h}$ and for a few at $24 \mathrm{~h}$, and additionally included aspartate, alanine, GPC and nucleotide sugars (UDP-glucose, UDPGalactose and UDP-GalNAc). Lower availability and only a transient increase after medium supplementation of alanine, glutamate, aspartate and taurine in ARR compared to SIL-TAL cells show that ARR have a high requirement for amino acids to facilitate higher rate of de novo protein synthesis (Figure 4C,D,2A,B). The SIL-TAL cells rapidly utilise essential amino acids upon medium change, followed by their intracellular accumulation. Altogether, measurements of the intracellular metabolite levels and the changes occurring during the cell growth and proliferation confirm that T-ALL have an unfavourable energy status with high AMP/ATP ratios and that SIL-TAL cells have limited availability of essential amino acids in line with the previously observed reduced de novo protein synthesis.

\section{Inhibition of the mTOR Pathway Restores Metabolite Levels in T-ALL Cell Lines}

As we showed that $\mathrm{mTOR}$ inhibition reduced glucose/glutamine uptake and blocked SIL-TAL proliferation (Figure 4B,2C), we next measured the effect of rapamycin on intracellular metabolite levels. NMR measurements identified 28 metabolites that had a significantly different abundance after $24 \mathrm{~h}$ rapamycin treatment in at least one of the cell lines (Figure $4 \mathrm{E}$ ). Hierarchical clustering of the observed log2 fold changes divided these metabolites into six rapamycin-responsive metabolic clusters (RMC1-6). The most pronounced effect of rapamycin was increased intracellular levels of the majority of metabolites. In all four cell lines the largest increase was observed for glutamine and alanine levels, while AMP and succinate levels had the opposite response (Figure 4E,S4B). A differential effect of rapamycin on SIL-TAL in comparison to ARR was particularly visible in RMC2 and RMC4. The levels of the amino acids phenylalanine, leucine, tyrosine, isoleucine and valine (RMC2) increased to a greater degree in SIL-TAL cells, whereas the increase in aspartate, glycine, glutamate and alanine (RMC4) was more pronounced in ARR (Figure 4E). The observed increase in the amino acids availability upon mTOR inhibition explains the mTOR recruitment to lysosomes upon rapamycin treatment and proves that T-ALL have limited amino acid availability. 


\section{Glutamine is Essential for SIL-TAL Proliferation and Survival and Fuels Aspartate and Nucleotide Biosynthesis}

The polarised effect of mTOR inhibition on ARR vs SIL-TAL, seen primarily as an increase in aspartate, glycine, glutamate and alanine levels could be due to the continuous proliferation in ARR and suppressed proliferation in SIL-TAL cells. As all four amino acids can be derived from glutamine and glutamine, glycine and aspartate are used as substrates for nucleotide synthesis, we tested the effect of glutamine withdrawal on cell proliferation. Omitting glutamine from the growth medium, while maintaining all other nutrients, including $10 \%$ foetal bovine serum, SIL-TAL cell numbers were reduced by $95 \%$ after 8 days of treatment (Figure 5A). Glutamine deprivation caused a decrease in ARR numbers over the first four days of culture, after which ARR recovered and resumed proliferation at the original rate. Apoptosis assays of the cells grown in glutamine-free medium revealed that the T-ALL cell lines had an increased rate of apoptosis (Figure S5). Therefore, we conclude that glutamine is important for T-ALL cell proliferation.

Glutamine has several significant roles in metabolic processes such as fuelling the TCA cycle through glutaminolysis and acting as a nitrogen donor in transamination and transamidation reactions that are used for the production of non-essential amino acids. Also, together with aspartate and glycine, glutamine feeds de novo nucleotide synthesis. In order to assess the glutamine contribution to T-ALL metabolic processes we performed tracer experiments using $\left[2,5-{ }^{15} \mathrm{~N}\right]$ Glutamine and $\left[3-{ }^{13} \mathrm{C}\right]$ Glutamine. When cells were grown in the presence of $\left[2,5-{ }^{15} \mathrm{~N}\right]$ Glutamine, in addition to the expected ${ }^{15} \mathrm{~N}$ labelled glutamate (data not shown), we observed ${ }^{15} \mathrm{~N}$ incorporation into the amino group of aspartate and alanine within $8 \mathrm{~h}$ of treatment in all four cell lines (Figure 5B and S6A). Resonances arising from $\mathrm{H} \beta \mathrm{s}$ of aspartate (2.80-2.81ppm) and alanine (1.48-1.49ppm) are shown. At Oh, a doublet was seen due to coupling to the $\mathrm{H} \alpha$. However, at later time points (Figure $5 \mathrm{~B}$, $8 \mathrm{~h}$ and $24 \mathrm{~h}$ ) there was increased spectral complexity due to weak coupling of $\mathrm{H} \beta \mathrm{s}$ to ${ }^{15} \mathrm{~N}$. The resulting spectrum was a weighted average of a doublet of doublets (from ${ }^{15} \mathrm{~N}$-labelled aspartate) and a simple doublet (unlabelled aspartate). This finding demonstrates that glutamine-derived ${ }^{15} \mathrm{~N}$ was incorporated into aspartate and alanine by transamination of oxaloacetate and pyruvate, respectively.

Glutamine, glycine and aspartate are amino acids used as substrates for nucleotide synthesis, so in order to assess the glutamine contribution to nucleotide biosynthesis we first acquired $2 \mathrm{D}-{ }^{1} \mathrm{H},{ }^{15} \mathrm{~N}$ heteronuclear single quantum coherence (HSQC) NMR spectra of ${ }^{15} \mathrm{~N}$ uniformly labelled ATP, GTP and UTP standards, where we observed five ${ }^{1} \mathrm{H}-{ }^{15} \mathrm{~N}$ interactions for $\left[\mathrm{U}-{ }^{15} \mathrm{~N}\right]$ ATP $(\mathrm{a}-\mathrm{d})$, three for $\left[\mathrm{U}-{ }^{15} \mathrm{~N}\right]$ GTP $(\mathrm{a}-\mathrm{c})$ and three for $\left[\mathrm{U}-{ }^{15} \mathrm{~N}\right]$ UTP $(\mathrm{a}-\mathrm{c})$ (Figure $5 \mathrm{C}$ ). Spectra acquired from $\left[2,5-{ }^{15} \mathrm{~N}\right]$ Glutamine-labelled T-ALL cells showed ${ }^{15} \mathrm{~N}$ 
incorporation into nucleotides at the 1, 3 and 9 positions, the 3 and 9 positions and the 1 and 3 positions in adenine, guanine and uracil respectively (Figure $5 \mathrm{C}$ ). As expected, based on the known contribution of glutamine to nucleotide synthesis, transamidation using ${ }^{15} \mathrm{~N}$ glutamine resulted in label incorporation at N-3 and N-9 in purines and the N-3 in pyrimidines (Supplementary Illustration SI1,2). However, label incorporation at $\mathrm{N}-1$ in purines and N-3 in pyrimidines is sourced from glutamine-derived ${ }^{15} \mathrm{~N}$-aspartate and shows that glutamine ultimately supplies all but one nitrogen in purine and both nitrogens in the pyrimidine ring.

So far, we demonstrated that glutamine transamination of oxaloacetate gives rise to aspartate. It is possible that glutamine supplies oxaloacetate, through glutaminolysis, which would consequently mean that aspartate is completely derived from glutamine. We used [3$\left.{ }^{13} \mathrm{C}\right]$ Glutamine in labelling experiments to test this. In the course of the TCA cycle, $\left[3-{ }^{13} \mathrm{C}\right]$ Glutamine is converted to $\left[2-{ }^{13} \mathrm{C}\right]$ Fumarate, a symmetrical molecule which is hydrated equally to $\left[2-{ }^{13} \mathrm{C}\right]$ Malate and $\left[3-{ }^{13} \mathrm{C}\right]$ Malate, and further to $\left[2-{ }^{13} \mathrm{C}\right]$ or $\left[3-{ }^{13} \mathrm{C}\right]$ oxaloacetate and aspartate (Supplementary Illustration SI3). Acquired $2 \mathrm{D}-{ }^{1} \mathrm{H},{ }^{13} \mathrm{C} \mathrm{HSQC}$ NMR spectra revealed ${ }^{13} \mathrm{C}$ incorporation in fumarate, malate, oxaloacetate and aspartate (Figure 5D,S6B and data not shown). Resonances for ${ }^{1} \mathrm{H}-{ }^{13} \mathrm{C}$ moieties were derived from both $\left[2-{ }^{13} \mathrm{C}\right]$ and $\left[3-{ }^{13} \mathrm{C}\right]$ labelling, as shown for aspartate and malate (a and b respectively, Fig 5D,S6B). Quantification of the signals from the labelled samples, relative to the naturally occurring ${ }^{13} \mathrm{C}$ in the control samples, showed that within $24 \mathrm{~h}, 50 \%$ of all aspartate was $\left[2-{ }^{13} \mathrm{C}\right]$ or $\left[3-{ }^{13} \mathrm{C}\right]$ Aspartate (Figure S6E). These findings confirmed our hypothesis that two molecules of glutamine give rise to a single aspartate molecule; first glutamine supplies the backbone via the TCA cycle, while the second is used for transamination.

A further implication of these findings was that carbons derived from glutamine would, via aspartate, get incorporated into pyrimidines, giving rise to $\left[5^{-13} \mathrm{C}\right]$ or $\left[6-{ }^{13} \mathrm{C}\right]$ Uridine (Supplementary illustration SI2). Resonances for ${ }^{1} \mathrm{H}-{ }^{13} \mathrm{C}$ moieties arising from $\left[5-{ }^{13} \mathrm{C}\right]$ Uridine or $\left[6-{ }^{13} \mathrm{C}\right]$ Uridine were indeed observed in $2 \mathrm{D}-{ }^{1} \mathrm{H},{ }^{13} \mathrm{C}$ HSQC NMR spectra (peaks a and $\mathrm{b}$, Figure 5D). This strongly implies that the carbon at position 4 also originates from glutamine via aspartate. Together, our results show that glutamine serves as a source for all but one of the atoms in the pyrimidine ring.

Examination of the ${ }^{13} \mathrm{C}$ incorporation in other detectable metabolites found a significant label accumulation into proline, as would be expected since glutamate is used as a substrate for proline synthesis (Figure S6C). Surprisingly, $\left[3-{ }^{13} \mathrm{C}\right]$ Proline was detected in all four cell lines, but $\left[2-{ }^{13} \mathrm{C}\right]$ Proline was found only in the SIL-TAL cells, indicating that SIL-TAL derived some glutamate from the TCA-cycle while ARR used glutamate almost exclusively derived directly from glutamine. Additional differences were observed between ARR and the SIL-TAL, in the area of the spectra that is characteristic for the aspartate-derived metabolites arginine, ornithine, citrulline and argininosuccinate $\left({ }^{13} \mathrm{C}-30.2-30.8 \mathrm{ppm}\right.$, Figure S6D). Due to overlaps 
between their spectra we were not able to identify the individual contributions of these metabolites. However, it is likely that the observed differences can be attributed to low argininosuccinate and arginine levels in SIL-TAL cells due to the finding that SIL-TAL cells lack ASS1 expression (Figure 1C,D), an enzyme essential for their biosynthesis.

\section{Patient-derived T-ALL and T-ALL cell lines have similar metabolic uptake}

To determine if glutamine is not only used by patient derived cell lines, but also by primary TALL patient isolates, we measured live-cell real-time metabolite uptake by NMR. T-ALL $\mathrm{CD}^{+} / \mathrm{CD}^{+}$cells were isolated from three T-ALL patients at presentation. The flow cytometry profile revealed that samples had cytoplasmic CD3 expression, but lacked CD3 surface expression. T-ALL_1 consisted of $60 \% \mathrm{CD} 34^{+}$blasts, half of which were $\mathrm{CD} 7^{+}$. TALL_2 was predominantly $\mathrm{CD}^{+}$, as well as $\mathrm{CD}^{+}, \mathrm{CD}^{+}, \mathrm{CD}^{+} 8^{+}$and $\mathrm{CD}^{+}$, whereas T-

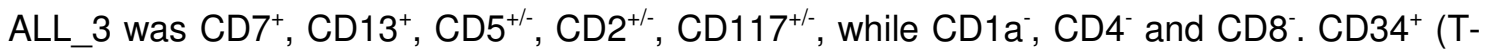
ALL_1) or $\mathrm{CD}^{+}$(T-ALL_2/3) cells were isolated and $10^{6}$ cells were resuspended in RPMI medium supplemented with GlutaMAX (L-glutamine/L-alanine dipeptide) and used for measuring metabolite uptake. GlutaMAX is a temperature-stable source of glutamine [53]. The L-Glutamine/L-Alanine dipeptide is hydrolysed, by peptidases located on the plasma membrane, into L-Glutamine and L-Alanine, which can then be taken up by the cells [54]. Therefore, the extracellular glutamine and alanine concentration depends on GlutaMAX hydrolysis and their utilisation by the cells. Using live-cell NMR we observed for all the patient samples that GlutaMAX concentrations decreased without the equivalent increase in glutamine availability (Figure 6A). Additionally, T-ALL_1 was the only one to take up glucose and pyruvate and releasing lactate.

\section{T-ALL cells express SLC1A3 and its protein product EAAT1 is localised in the mitochondria}

We demonstrated that the contribution of glutamine, directly and through aspartate, is crucial for T-ALL nucleotide synthesis and proliferation. As glutamine uptake and its direct contribution to nucleotide metabolism and TCA cycle is indispensable for whole organism survival, we focused on identifying a useful therapeutic target for T-ALL treatment on metabolic processes supporting glutamine-derived aspartate synthesis (Supplementary Illustration SI3). Inhibiting the glutamate-aspartate anti-port across the mitochondrial membrane would be a good strategy for targeting T-ALL as it is not directed towards the enzymatic conversions. Aspartate export and glutamate import into mitochondria is known to be facilitated by 3 different antiporters SLC1A3, SLC25A12 and SLC25A13 [55, 56]. While SLC25A12 and SLC25A13 are expressed in many tissues, the expression of SLC1A3 gene is mainly restricted to CNS [57]. Analyses of our RNAseq data, as well as recently published 
gene expression data of $265 \mathrm{~T}$-ALL patient samples, revealed that $S L C 1 A 3$ is expressed in our model cell lines and in 95\% of patient samples (Figure 6B,C)[8]. Additionally, we observed that EAAT1, the protein encoded by SLC1A3, was detected in the mitochondria of the T-ALL cell lines. SLC1A3/EAAT1 was not detected in the acute myeloid leukaemia (AML) cell line Kasumi-1, which does not express SLC1A3 (Figure 6D,E) [58]. Together, these results show that T-ALL express the high affinity antiporter EAAT1.

\section{SLC1A3/EAAT1 is essential for oncogenic de novo nucleotide synthesis}

The mitochondrial localisation of EAAT1 in T-ALL cells supports the possibility that this protein is involved in an oncogenic glutamate-aspartate antiport in T-ALL and crucial for TALL proliferation and survival. In order to assess the importance of EAAT1 for T-ALL survival we performed SLC1A3 knock-down using shRNA. Five shSLC1A3 were designed and tested for their capacity to supresses the expression of SLC1A3 cDNA. Mouse fibroblasts were co-transduced with retrovirus expressing SLC1A3-IRES-GFP and shSLC1A3. The efficiency of the shRNA was measured relative to the negative control, shFF3, which targets firefly luciferase and the positive control, shGFP, that supresses GFP expression originating from the second cistron of the SLC1A3-IRES-GFP mRNA. shSLC1A3_1 and particularly _2 had the capacity to supress EAAT1 protein levels similar to shGFP, while the negative control shFF3 did not have any effect on EAAT1 protein levels (Figure 7A). shSLC1A3_1 and _2 were cloned into a PiggyBac backbone that supports doxycycline-inducible shRNA expression and integrated into the T-ALL cell genomes. Induction of shSLC1A3 lead to rapid cell death in ARR, DU.528 and CCRF_CEM cells within 5 days of the doxycycline treatment (Figure 7B). We were unable to produce the same results with HSB2 cells since these cells were affected by doxycycline treatment alone. These results corroborate that SLC1A3/EAAT1 is required for T-ALL cell proliferation and survival.

In healthy adults EAAT1 is normally expressed in the CNS $[18,57]$. With the purpose to inhibit its function in the CNS, two specific allosteric inhibitors, UCPH-101 and UCPH-102 were developed $[59,60]$. Both drugs exhibited inhibitory effects on T-ALL proliferation and, while UCPH-101 had a cytotoxic effect that reduced the survival of the control AML cell line Kasumi-1, UCPH-102 had a specific anti-proliferative effect only on T-ALL cells (Figure 7C). Cytotoxicity connected with UCPH-101 can be explained by the presence of known toxicophores. Taken together, SLC1A3 knock-down and pharmacological inhibition caused the supression of T-ALL proliferation. This effect is similar to the effect of glutamine removal from the growth medium (Figure $5 \mathrm{~A}$ ). Finally, in order to confirm the mechanism of UCPH102 mediated SLC1A3 inhibition, we measured the metabolite uptake/release. UCPH-102 abolished glutamine uptake in ARR cells, while statistically significant changes in all four TALL cell lines were only observed for glutamate and aspartate (Figure 7D and S7B). EAAT1 
inhibition increased glutamate release in the ARR cell culture and decrease in the others, while aspartate was reduced in the growth medium of all four cell lines (Figure 7D). Reduced apartate release into the medium implies limited intracellular availability of aspartate that would impact on nucleotide synthesis, hence T-ALL proliferation. This corroborates our finding that EAAT1 is required for T-ALL survival and proliferation.

\section{Development of EAAT1 inhibitors to block T-ALL cell proliferation}

$\mathrm{UCPH}-101$ and UCPH-102 were developed to cross the blood-brain barrier with the aim to reach the CNS. Despite extensive development from the original hit compounds, these two compounds have many unfavourable characteristics, including toxicophores and a very short half life in vivo [61]. We therefore set out to identify new small molecule inhibitors of EAAT1 with potential to be be further developed for T-ALL treatment. As the UCPH compounds show high specificity due to their allosteric inhibitory function, an in silico approach was chosen on basis of the available crystal structure of EAAT1 in complex with UCPH-101 [60]. The in silico screen was performed by Domainex Ltd, using their database of approximately 2 million commercially available compounds with lead-like characteristics. Compounds were selected that have the potential to interact at the same position as $\mathrm{UCPH}-101$ and were screened in an in vitro cell assay on T-ALL cell lines and the Kasumi-1 cell line as a negative control. This cellular proliferation screen resulted in compounds that supressed the growth of T-ALLs when compared to Kasumi-1. EAAT1inh_1 showed a significant effect at concentrations below $1 \mu \mathrm{M}$ (Figure 7E).

\section{Discussion}

Describing metabolic pathways is pivotal for understanding the processes that are essential for embryonic development, homeostasis and disease progression. Metabolic processes are interconnected and many steps are supported by several different enzymes that can be located in different cellular compartments and even different tissues and organs. Understanding the capacity of the cells to utilise a certain metabolic pathway based on the gene expression and combining this with the measurements of cellular demand and output is essential for building a bigger picture of the metabolic processes that support cellular proliferation and survival. One of the main characteristics of cancer cells is increased proliferation. During the oncogenic transformation, changes in gene expression and cellular signalling allow uncontrolled proliferation. In order to successfully achieve this, cancer cells may use processes that are more commonly observed during embryonic development, when large quantities of nutrients are used for growth and organogenesis. Understanding the 
metabolic pathways that support oncogenic proliferation can help us identify cancer-specific processes and rate-limiting steps that can be used for developing new therapies.

Our results demonstrate that integration of gene expression, signal transduction and metabolic studies provides a powerful approach for the dissection of key metabolic processes. We show that, in T-ALL cells, mTOR regulates glutamine uptake. Glutamine is converted to glutamate that enters the mitochondria where Glutamate Dehydrogenase (GLUD1,2) uses it to generate $\alpha$-ketoglutarate that will enter the TCA cycle (Figure 7F). Additionally, mitochondrial Glutamate Oxaloacetate Transaminase (GOT2) uses glutamate as a donor for the amino group, which is transferred to oxaloacetate, another TCA cycle intermediate, resulting in generation of aspartate and a-ketoglutarate. Aspartate is then transported out of the mitochondria in exchange for a new glutamate molecule, continuing to fuel the TCA cycle and generating aspartate. Together, glutamine and aspartate are used as substrates for nucleotide synthesis (Figure 7F). Similar processes are likely to occur in other cancers. Indeed, recently EAAT1 has been implicated in supporting proliferation in several cancer cell lines representing solid cancers $[62,63]$. Both studies showed a main role for EAAT1 in the uptake of aspartate from the medium, especially under conditions of glutamine deprivation or asparaginase treatment. In both studies removing EAAT1 under normal cell culture conditions showed little or no effect in contrast to our work on T-ALL cells. This difference can be explained as the solid tumour cell lines were mainly dependent on EAAT1 for aspartate/glutamate uptake from the environment and the lack thereof resulted in a combinatorial effect on the TCA cycle, the electron transport chain, and de novo glutamine/glutamate and nucleotide synthesis. T-ALL cells on the other hand are dependent on the availability of glutamine. They rely heavily on the function of EAAT1 on the inner membrane of the mitochondria, where glutamate/aspartate antiport function is required for de novo nucleotide production. This process is heavily dependent on the availability of glutamate, which is derived from glutamine. Our NMR tracer experiments show that the carbon and nitrogen atoms of glutamine are used for aspartate, purine and pyrimidine biosynthesis. Sun et al. also showed that asparaginase treatment resulted in a cellular depletion of glutamine/glutamate, possibly explaining why asparaginase treatment is often effective in treatment of T-ALL [64].

Systemic targeting of the metabolic enzymes that conduct the glutamine to aspartate conversion or nucleotide synthesis would be detrimental. Our finding that the transport of aspartate to the cytoplasm is an essential step in nucleotide production and that it is facilitated by aberrantly expressed EAAT1, identifies this protein as a novel therapeutic target for developing treatments for T-ALL. EAAT1 is normally present in the CNS on the plasma membrane of neurons and glia where it uptakes glutamate from the glutamatergic synapses [17]. Based on the RNA expression and protein localisation assessed with three 
different specific antibodies, the Human Protein atlas Database reports that EAAT1 is not found outside of the CNS (https://www.proteinatlas.org/ENSG00000079215-SLC1A3/tissue) [65]. However, EAAT1 expression has been reported in neonatal cardiomyocytes where, similar to T-ALL, it is also localised in mitochondria together with two other glutamateaspartate antiporters, i.e. ARALAR1 and CITRIN [55]. ARALAR1 and CITRIN are calciumbinding mitochondrial carrier proteins that import a glutamate molecule together with $\mathrm{a} \mathrm{H}^{+}$ into the mitochondria in exchange for the export of an aspartate anion [66-68]. Their activity depends on the mitochondrial membrane potential that is maintained by cytoplasmic ATP [69]. Both transporters are present in the majority of cells and tissues (reviewed in [70]). We found that SLC25A12 is expressed in all four T-ALL cell lines, while SLC25A13 was found only in DU.528. With this in mind, an obvious question is why mitochondrial EAAT1 is necessary for T-ALL survival. One possibility is that EAAT1 has a higher Kd for glutamate than ARALAR1and CITRIN and therefore facilitates a higher rate of antiport across the mitochondrial membrane. Even though the kinetics of all three transporters are reported, the experiments were not performed in comparable experimental conditions and EAAT1 capacity was always addressed in respect to its membranous localisation [70-72]. The second possibility is that the intracellular conditions, such as the $\mathrm{pH}$, ATP availability and mitochondrial action potential, are restrictive for ARALAR1 and CITRIN function, whereas EAAT1 is independent of these factors. Altogether, this means that the aberrant expression of SLC1A3 in T-ALL renders this gene as an oncogene and EAAT1 a therapeutic target. We therefore performed a screen for novel small molecule inhibitors and we present EAAT1inh_1 compound as a potent EAAT1 inhibitor.

\section{Acknowledgments}

We would like to thank Dr. A.W. Langerak, (Erasmus Medical Centre, Rotterdam, NL) for the provision of ARR and DU.528 cell lines and Prof P.N. Cockerill (University of Birmingham, UK) for HSB2 and CCRF-CEM. We would like to thank Dr. M. McGrew (University of Edinburgh, UK) for the PB_tet-on_Apple_shGFP plasmid and Prof. L. Bunch for pcDNA3EAAT1 plasmid. We would like to acknowledge BlueBEAR High Performance Computing (HPC) service for supporting the analyses of the genome-wide data and Biomolecular NMR Facility at the Henry Wellcome Building for Nuclear Magnetic Resonance (HWB-NMR), University of Birmingham. This work was supported by Bloodwise, through a Bennett Fellowship to M.H. [11002], the Medical Research Council and the University of Birmingham.

\section{Authorship Contributions}

Original Concept V.S.S., Project Planning, Experimental Design, Project supervision, V.S.S., M.H.; Genome-wide data acquisition and Bioinformatical analyses V.S.S., E.G., H.P., M.H.; 
Statistical analyses V.S.S.; NMR data acquisition and analyses M.A.C.R, J.R., V.S.S, U.G., C.L.; Drug screen V.S.S.; Consultations on chemical matter A.M.J.; All other Experimentation, Data Acquisition, Processing and Analyses V.S.S., M.H.; Provision of patient samples S.P., G.P.; Reagents provision S.S.; Figures V.S.S.; Writing of the Manuscript, V.S.S., M.A.C.R. and M.H. 
1. Nagarajan, A., P. Malvi, and N. Wajapeyee, Oncogene-directed alterations in cancer cell metabolism. Trends Cancer, 2016. 2(7): p. 365-377.

2. Shoshan, M., On mitochondrial metabolism in tumor biology. Curr Opin Oncol, 2017. 29(1): p. 48-54.

3. Tennant, D.A., R.V. Duran, and E. Gottlieb, Targeting metabolic transformation for cancer therapy. Nat Rev Cancer, 2010. 10(4): p. 267-77.

4. Mossmann, D., S. Park, and M.N. Hall, mTOR signalling and cellular metabolism are mutual determinants in cancer. Nat Rev Cancer, 2018. 18(12): p. 744-757.

5. Fruman, D.A. and C. Rommel, PI3K and cancer: lessons, challenges and opportunities. Nat Rev Drug Discov, 2014. 13(2): p. 140-56.

6. Mayer, I.A. and C.L. Arteaga, The PI3K/AKT Pathway as a Target for Cancer Treatment. Annu Rev Med, 2016. 67: p. 11-28.

7. Homminga, I., et al., Integrated transcript and genome analyses reveal NKX2-1 and MEF2C as potential oncogenes in T cell acute lymphoblastic leukemia. Cancer Cell, 2011. 19(4): p. 48497.

8. Liu, Y., et al., The genomic landscape of pediatric and young adult T-lineage acute lymphoblastic leukemia. Nat Genet, 2017. 49(8): p. 1211-1218.

9. Vicente, C., et al., Targeted sequencing identifies associations between IL7R-JAK mutations and epigenetic modulators in T-cell acute lymphoblastic leukemia. Haematologica, 2015. 100(10): p. 1301-10.

10. Kataoka, K., et al., Integrated molecular analysis of adult $T$ cell leukemia/lymphoma. Nat Genet, 2015. 47(11): p. 1304-15.

11. Vora, A., et al., Treatment reduction for children and young adults with low-risk acute lymphoblastic leukaemia defined by minimal residual disease (UKALL 2003): a randomised controlled trial. Lancet Oncol, 2013. 14(3): p. 199-209.

12. Chessells, J.M., et al., Long-term follow-up of relapsed childhood acute lymphoblastic leukaemia. Br J Haematol, 2003. 123(3): p. 396-405.

13. Ajay Vora, R.W., Christopher D Mitchell, Nicholas Goulden and Sue Richards, Improved Outcome for Children and Young Adults with T-Cell Acute Lymphoblastic Leukaemia (ALL): Results of the United Kingdom Medical Research Council (MRC) Trial UKALL 2003. Blood, 2008. 112(11): p. 908.

14. Hastings, C., et al., Increased post-induction intensification improves outcome in children and adolescents with a markedly elevated white blood cell count (>/=200 x 10(9) /I) with T cell acute lymphoblastic leukaemia but not $B$ cell disease: a report from the Children's Oncology Group. Br J Haematol, 2015. 168(4): p. 533-46.

15. Vora, A., et al., Augmented post-remission therapy for a minimal residual disease-defined high-risk subgroup of children and young people with clinical standard-risk and intermediaterisk acute lymphoblastic leukaemia (UKALL 2003): a randomised controlled trial. Lancet Oncol, 2014. 15(8): p. 809-18.

16. Aplan, P.D., et al., Involvement of the putative hematopoietic transcription factor SCL in T-cell acute lymphoblastic leukemia. Blood, 1992. 79(5): p. 1327-33.

17. Lehre, K.P., et al., Differential expression of two glial glutamate transporters in the rat brain: quantitative and immunocytochemical observations. J Neurosci, 1995. 15(3 Pt 1): p. 1835-53.

18. Storck, T., et al., Structure, expression, and functional analysis of a $\mathrm{Na}(+)$-dependent glutamate/aspartate transporter from rat brain. Proc Natl Acad Sci U S A, 1992. 89(22): p. 10955-9.

19. Sandberg, Y., et al., Human T-cell lines with well-defined T-cell receptor gene rearrangements as controls for the BIOMED-2 multiplex polymerase chain reaction tubes. Leukemia, 2007. 21(2): p. 230-7. 
20. Begley, C.G., et al., Chromosomal translocation in a human leukemic stem-cell line disrupts the $T$-cell antigen receptor delta-chain diversity region and results in a previously unreported fusion transcript. Proc Natl Acad Sci U S A, 1989. 86(6): p. 2031-5.

21. Adams, R.A., A. Flowers, and B.J. Davis, Direct implantation and serial transplantation of human acute lymphoblastic leukemia in hamsters, SB-2. Cancer Res, 1968. 28(6): p. 1121-5.

22. Aplan, P.D., et al., Disruption of the human SCL locus by "illegitimate" V-(D)-J recombinase activity. Science, 1990. 250(4986): p. 1426-9.

23. Foley, G.E., et al., Continuous Culture of Human Lymphoblasts from Peripheral Blood of a Child with Acute Leukemia. Cancer, 1965. 18: p. 522-9.

24. Chou, T.C. and F. Lipmann, Separation of acetyl transfer enzymes in pigeon liver extract. J Biol Chem, 1952. 196(1): p. 89-103.

25. Hele, P., The acetate activating enzyme of beef heart. J Biol Chem, 1954. 206(2): p. 671-6.

26. Ratner, S., Urea synthesis and metabolism of arginine and citrulline. Adv Enzymol Relat Subj Biochem, 1954. 15: p. 319-87.

27. Liu, J., et al., Imaging protein synthesis in cells and tissues with an alkyne analog of puromycin. Proc Natl Acad Sci U S A, 2012. 109(2): p. 413-8.

28. Yang, Q. and K.L. Guan, Expanding mTOR signaling. Cell Res, 2007. 17(8): p. 666-81.

29. Hara, K., et al., Amino acid sufficiency and mTOR regulate $p 70$ S6 kinase and elF-4E BP1 through a common effector mechanism. J Biol Chem, 1998. 273(23): p. 14484-94.

30. Dennis, P.B., et al., Mammalian TOR: a homeostatic ATP sensor. Science, 2001. 294(5544): p. 1102-5.

31. Heitman, J., N.R. Movva, and M.N. Hall, Targets for cell cycle arrest by the immunosuppressant rapamycin in yeast. Science, 1991. 253(5022): p. 905-9.

32. Kunz, J., et al., Target of rapamycin in yeast, TOR2, is an essential phosphatidylinositol kinase homolog required for $G 1$ progression. Cell, 1993. 73(3): p. 585-96.

33. Kim, E., et al., Regulation of TORC1 by Rag GTPases in nutrient response. Nat Cell Biol, 2008. 10(8): p. 935-45.

34. Sancak, Y., et al., The Rag GTPases bind raptor and mediate amino acid signaling to mTORC1. Science, 2008. 320(5882): p. 1496-501.

35. Sancak, Y., et al., Ragulator-Rag complex targets mTORC1 to the lysosomal surface and is necessary for its activation by amino acids. Cell, 2010. 141(2): p. 290-303.

36. Ho, M.K. and T.A. Springer, Tissue distribution, structural characterization, and biosynthesis of Mac-3, a macrophage surface glycoprotein exhibiting molecular weight heterogeneity. J Biol Chem, 1983. 258(1): p. 636-42.

37. Marsh, M., et al., Rapid analytical and preparative isolation of functional endosomes by free flow electrophoresis. J Cell Biol, 1987. 104(4): p. 875-86.

38. Chazotte, B., Labeling lysosomes in live cells with LysoTracker. Cold Spring Harb Protoc, 2011. 2011(2): p. pdb prot5571.

39. Hara, K., et al., Raptor, a binding partner of target of rapamycin (TOR), mediates TOR action. Cell, 2002. 110(2): p. 177-89.

40. Kim, D.H., et al., mTOR interacts with raptor to form a nutrient-sensitive complex that signals to the cell growth machinery. Cell, 2002. 110(2): p. 163-75.

41. Beugnet, A., X. Wang, and C.G. Proud, Target of rapamycin (TOR)-signaling and RAIP motifs play distinct roles in the mammalian TOR-dependent phosphorylation of initiation factor $4 E-$ binding protein 1. J Biol Chem, 2003. 278(42): p. 40717-22.

42. Nojima, H., et al., The mammalian target of rapamycin (mTOR) partner, raptor, binds the mTOR substrates $p 70$ S6 kinase and 4E-BP1 through their TOR signaling (TOS) motif. J Biol Chem, 2003. 278(18): p. 15461-4.

43. Gwinn, D.M., et al., AMPK phosphorylation of raptor mediates a metabolic checkpoint. Mol Cell, 2008. 30(2): p. 214-26. 
44. Hawley, S.A., et al., Characterization of the AMP-activated protein kinase kinase from rat liver and identification of threonine 172 as the major site at which it phosphorylates AMPactivated protein kinase. J Biol Chem, 1996. 271(44): p. 27879-87.

45. Lizcano, J.M., et al., LKB1 is a master kinase that activates 13 kinases of the AMPK subfamily, including MARK/PAR-1. EMBO J, 2004. 23(4): p. 833-43.

46. Shaw, R.J., et al., The tumor suppressor LKB1 kinase directly activates AMP-activated kinase and regulates apoptosis in response to energy stress. Proc Natl Acad Sci U S A, 2004. 101(10): p. 3329-35.

47. Mao, K. and D.J. Klionsky, AMPK activates autophagy by phosphorylating ULK1. Circ Res, 2011. 108(7): p. 787-8.

48. Kim, J., et al., Differential regulation of distinct Vps34 complexes by AMPK in nutrient stress and autophagy. Cell, 2013. 152(1-2): p. 290-303.

49. Chan, E.Y., S. Kir, and S.A. Tooze, siRNA screening of the kinome identifies ULK1 as a multidomain modulator of autophagy. J Biol Chem, 2007. 282(35): p. 25464-74.

50. Liang, X.H., et al., Induction of autophagy and inhibition of tumorigenesis by beclin 1. Nature, 1999. 402(6762): p. 672-6.

51. Ktistakis, N.T. and S.A. Tooze, Digesting the Expanding Mechanisms of Autophagy. Trends Cell Biol, 2016. 26(8): p. 624-635.

52. Zhou, G., et al., Role of AMP-activated protein kinase in mechanism of metformin action. J Clin Invest, 2001. 108(8): p. 1167-74.

53. Roth, E., et al., Influence of two glutamine-containing dipeptides on growth of mammalian cells. In Vitro Cell Dev Biol, 1988. 24(7): p. 696-8.

54. Christie, A. and M. Butler, Glutamine-based dipeptides are utilized in mammalian cell culture by extracellular hydrolysis catalyzed by a specific peptidase. J Biotechnol, 1994. 37(3): p. 27790.

55. Ralphe, J.C., et al., Localization and function of the brain excitatory amino acid transporter type 1 in cardiac mitochondria. J Mol Cell Cardiol, 2004. 37(1): p. 33-41.

56. Lasorsa, F.M., et al., Recombinant expression of the Ca(2+)-sensitive aspartate/glutamate carrier increases mitochondrial ATP production in agonist-stimulated Chinese hamster ovary cells. J Biol Chem, 2003. 278(40): p. 38686-92.

57. Fagerberg, L., et al., Analysis of the human tissue-specific expression by genome-wide integration of transcriptomics and antibody-based proteomics. Mol Cell Proteomics, 2014. 13(2): p. 397-406.

58. Ptasinska, A., et al., Identification of a dynamic core transcriptional network in $t(8 ; 21) A M L$ that regulates differentiation block and self-renewal. Cell Rep, 2014. 8(6): p. 1974-1988.

59. Erichsen, M.N., et al., Structure-activity relationship study of first selective inhibitor of excitatory amino acid transporter subtype 1: 2-Amino-4-(4-methoxyphenyl)-7-(naphthalen-1yl)-5-oxo-5,6,7,8-tetrahydro-4H-chrom ene-3-carbonitrile (UCPH-101). J Med Chem, 2010. 53(19): p. 7180-91.

60. Canul-Tec, J.C., et al., Structure and allosteric inhibition of excitatory amino acid transporter 1. Nature, 2017. 544(7651): p. 446-451.

61. Haym, I., et al., Bioavailability Studies and in vitro Profiling of the Selective Excitatory Amino Acid Transporter Subtype 1 (EAAT1) Inhibitor UCPH-102. ChemMedChem, 2016. 11(4): p. 403-19.

62. Sun, J., et al., SLC1A3 contributes to L-asparaginase resistance in solid tumors. EMBO J, 2019. 38(21): p. e102147.

63. Tajan, M., et al., A Role for $p 53$ in the Adaptation to Glutamine Starvation through the Expression of SLC1A3. Cell Metab, 2018. 28(5): p. 721-736 e6.

64. Pui, C.H., et al., Treating childhood acute lymphoblastic leukemia without cranial irradiation. N Engl J Med, 2009. 360(26): p. 2730-41. 
65. Uhlen, M., et al., Proteomics. Tissue-based map of the human proteome. Science, 2015. 347(6220): p. 1260419.

66. Thangaratnarajah, C., J.J. Ruprecht, and E.R. Kunji, Calcium-induced conformational changes of the regulatory domain of human mitochondrial aspartate/glutamate carriers. Nat Commun, 2014. 5: p. 5491.

67. Palmieri, L., et al., Citrin and aralar1 are $\mathrm{Ca}(2+)$-stimulated aspartate/glutamate transporters in mitochondria. EMBO J, 2001. 20(18): p. 5060-9.

68. LaNoue, K.F. and M.E. Tischler, Electrogenic characteristics of the mitochondrial glutamateaspartate antiporter. J Biol Chem, 1974. 249(23): p. 7522-8.

69. LaNoue, K.F., J. Bryla, and D.J. Bassett, Energy-driven aspartate efflux from heart and liver mitochondria. J Biol Chem, 1974. 249(23): p. 7514-21.

70. Amoedo, N.D., et al., AGC1/2, the mitochondrial aspartate-glutamate carriers. Biochim Biophys Acta, 2016. 1863(10): p. 2394-412.

71. Arriza, J.L., et al., Functional comparisons of three glutamate transporter subtypes cloned from human motor cortex. J Neurosci, 1994. 14(9): p. 5559-69.

72. Jensen, A.A. and H. Brauner-Osborne, Pharmacological characterization of human excitatory amino acid transporters EAAT1, EAAT2 and EAAT3 in a fluorescence-based membrane potential assay. Biochem Pharmacol, 2004. 67(11): p. 2115-27. 


\section{Figure Legends}

Figure 1. Differential gene expression and gene ontology analysis for T-ALL cell lines. A) Heat map showing hierarchical clustering of RNAseq gene expression data, based on Pearson correlation with complete linkage clustering of all differentially expressed genes between the T-ALL cell lines. Clusters are marked by blue triangles and numbered from 1 to 11. Scale bar represents log2 FPKM values. B) Gene ontology enrichment analysis for cluster 1, 9 and 10. Terms are ordered based on Modified Fisher Extract P-value and shown as percentage of input genes (\% genes). C) UCSC genome browser screenshots showing the distribution of reads across the ASS1 and ACSS1 from a representative set of RNAseq tracks. D) Validation of RNAseq results by qPCR, relative to rRNA levels. Data points are the mean of at least three independent samples measured in duplicate \pm StDev.

Figure 2. T-ALL cell lines have aberrant mTOR signalling. A) Protein synthesis was assessed by Click-iT OPP protein synthesis kit and imaged by confocal microscopy. Representative images are shown. B) Quantification of protein synthesis using CellProfiler software in arbitrary units [AU]. C) Rapamycin inhibits SIL-TAL proliferation. Cell cultures were set up in the presence of 10nM rapamycin or vehicle (DMSO) and cultured for 4 days. Four independent cell cultures were assayed per cell line and each point represents the mean \pm StDev. Significant differences were found when ARR was compared to any of the SIL-TAL cell lines at day 3 and $4 \quad(p<0.05)$. D) Confocal microscopy images of immunofluorescent staining for mTOR (green), LAMP1 (red), LysoTracker (grey), DAPI (blue) shows the nuclei. The overlay panel shows mTOR/LAMP1 localisation. E) Protein levels and phosphorylation status of mTOR regulator Raptor. F) Protein levels and phosphorylation status of AMPK and its targets Beclin and ULK1. Western blot analysis of $150 \mu \mathrm{g}$ cell extracts. Treatment was DMSO (-,Ctrl) or 10nM rapamycin (+,Rap) for $24 \mathrm{~h}$. Ponceaus and actin staining was used to confirm equal loading. Each experiment was performed at least three times and representative results are shown.

Figure 3. T-ALL cell lines have constitutively active AMPK that inhibits autophagy and Iysosomal function in SIL-TAL. A) Confocal microscopy images illustrate LC3 (green) and LAMP1 (red) distribution. DAPI (blue) shows the nuclei. Boxed area from the overlay image is enlarged in inset. B) p62 and LC3 protein levels. C) Confocal images of the LC3 (green), LysoTraker (red), LAMP1 (magenta) immunostainings. D) p62 and LC3 protein levels. Treatment was A,B) $24 \mathrm{~h} 10 \mathrm{nM}$ rapamycin (+,Rap) or C,D) $4 \mathrm{~h} 5 \mu \mathrm{M}$ dorsomorphine (+,D). Control was DMSO (-,Ctrl). PonceauS was used to confirm equal loading. Experiments were performed at least three times and representative results are shown. 
Figure 4. Metabolite levels in T-ALL cells are dynamic and sensitive to mTOR activity. A) Heat map showing hierarchical clustering of the growth medium metabolites after $24 \mathrm{~h}$. Data are the average of four independent experiments \pm StDev. Scale bar represents log2 relative metabolite concentration. Metabolites with significantly different levels are shown. $\mathbf{B}$ ) Rapamycin changes metabolite uptake and release. T-ALL cell lines were grown in the presence of rapamycin or vehicle (DMSO) for $24 \mathrm{~h}$. Relative metabolite levels are the mean of four independent experiments. Two-tailed t-test identified the difference in glutamine, glucose and lactate concentration as significantly different with $p<0.05$ between the cells grown with vehicle to rapamycin in all four cell lines. C) Heat map showing hierarchical clustering of intracellular metabolite levels after culturing T-ALL cells for $24 \mathrm{~h}$. Data are the average of at least three independent experimental measurements \pm StDev. Four different metabolite clusters (MC1-4) were identified. Scale bar represents log2 metabolite levels. Only metabolites with significantly different levels between at least two of the cell lines are shown. D) Abundance of intracellular metabolites at $8 \mathrm{~h}$ or $24 \mathrm{~h}$, relative to the time of medium change (0h). Fold change was presented only for metabolites with significantly different relative levels with $p<0.05$. E) Heat map showing hierarchical clustering of the log 2 ratio between metabolite levels in rapamycin treated cells and the control. Metabolites were isolated after culturing T-ALL cells for $24 \mathrm{~h}$ in the presence of $10 \mathrm{nM}$ rapamycin or vehicle (DMSO) from at least three independent samples. Six different metabolite clusters are labelled (RMC1-6). Scale bar represents log2 ratio between rapamycin-treated cells and the control. Only metabolites with significantly different levels are shown. GPC, L-Alpha glycerylphosphorylcholine; GalNAc, Acetylgalactosamine, GlcNAc, N-Acetylglucosamine.

Figure 5. T-ALL cells utilise glutamine-derived nitrogen and carbon for de novo nucleotide synthesis. A) Glutamine deprivation inhibits SIL-TAL proliferation. T-ALL cell lines were cultured in medium with $10 \%$ FCS, with or without $2 \mathrm{mM}$ GlutaMax. Four independent cell cultures were assayed per cell line and each point represents the mean \pm StDev. Significant differences were found when ARR was compared to any of the SIL-TAL cell lines at day 6 and $8(p<0.05)$. B-D) Metabolite tracing experiments using $\left[2,5-{ }^{15} \mathrm{~N}\right]$ Glutamine and $\left[3-{ }^{13} \mathrm{C}\right]$ Glutamine. T-ALL cell lines were grown in the presence of $2 \mathrm{mM}[2,5-$ $\left.{ }^{15} \mathrm{~N}\right]$ Glutamine) $(\mathrm{B}, \mathrm{C})$ or $\left[3^{-13} \mathrm{C}\right]$ Glutamine) (D). B) Overlay of $1 \mathrm{D}{ }^{1} \mathrm{H}$-NMR spectra showing the $\mathrm{H} \beta$-aspartate resonance after 0,8 and $24 \mathrm{~h}$ and schematic representations of $\left[2,5-{ }^{15} \mathrm{~N}\right]$ Glutamine and the observed $\left[2-{ }^{15} \mathrm{~N}\right]$ Aspartate. ${ }^{15} \mathrm{~N}$ are in red and shading indicates the observed ${ }^{3} \mathrm{~J}$ scalar couplings between the aspartate $\mathrm{H} \beta$ s and glutamine-derived ${ }^{15} \mathrm{~N}$. The $\mathrm{X}$ axis shows the chemical shift relative to TMSP in ppm and the $\mathrm{Y}$-axis indicates TSA scaled intensity. C) Resonances observed in ${ }^{1} \mathrm{H}-{ }^{15} \mathrm{~N}-\mathrm{HSQC}$ for $\left[\mathrm{U}-{ }^{15} \mathrm{~N}\right] \mathrm{ATP},\left[\mathrm{U}-{ }^{15} \mathrm{~N}\right] \mathrm{GTP},\left[\mathrm{U}-{ }^{15} \mathrm{~N}\right.$ ] 
UTP standards and for T-ALL cells extracts grown in the presence of Glutamine- $-{ }^{15} \mathrm{~N}_{2}$ for $24 \mathrm{~h}$. Resonances are marked by letters a-e. Schematics show ATP, GTP and UTP with colourcoded atoms based on the substrate of their origin (glutamine-purple, aspartate-orange, glycine-green, carbonate-black and ${ }^{15} \mathrm{~N}$-red). Blue shaded lines indicate observed couplings annotated a-e. D) Resonances observed in ${ }^{1} \mathrm{H}-{ }^{13} \mathrm{C}-\mathrm{HSQC}$ for T-ALL cells grown in the presence of [3-13C] Glutamine for 24h. Resonances are marked by letters a-e. Schematic on the right shows aspartate and UTP with colour-coded atoms based on the substrate of their origin. Blue shaded lines indicate observed couplings annotated a-e.

Figure 6. EAAT1 is expressed in T-ALL and localised in mitochondria. A) Patient derived T-ALL cells have similar metabolic uptake to T-ALL cell lines. Only metabolites with changing concentration are illustrated. B) Summary of the SLC1A3 expression levels as assessed by FPKM values from the RNA-seq of 265 patient samples. Data are grouped by the level of expression. C) SLC1A3 mRNA expression level in T-ALL cell lines and AML cell line Kasumi-1, relative to rRNA level assessed by qPCR. D) Western blot analysis of EAAT1 protein level using $150 \mu \mathrm{g}$ total cell extract. PonceauS shows equal loading. E) Immunofluorescent imaging shows that EAAT1 (green) co-localises with MitoTracker Red CMXRos in T-ALL but not Kasumi-1.

Figure 7. EAAT1 is essential for T-ALL proliferation and survival. A) Protein level of EAAT1 and the reporter GFP protein upon the suppression of SLC1A3-IRES-GFP mRNA by shFF3 (negative control), shGFP (positive control) and five different shSLC1A3_1-5. Experiment was performed in duplicate. PonceauS illustrates equal loading. B) Knock-down of the SLC1A3 gene by shSLC1A3_1 and shSLC1A3_2 leads to ARR, DU.528 and CCRF_M cell death. C) To test the effect of EAAT1 inhibition, T-ALL and AML cells were cultured for six days in the presence of vehicle (DMSO), $25 \mu \mathrm{M} U \mathrm{CPH}-101$, or $25 \mu \mathrm{M} U \mathrm{CPH}$ 102. Each data point is an average of three independent measurements \pm StDev. D) UCPH102 induced changes in metabolite uptake and release. T-ALL cell lines were grown in the presence of $25 \mu \mathrm{M}$ or vehicle (DMSO) for $48 \mathrm{~h}$. Relative metabolite levels are the mean of four independent experiments. Two-tailed t-test identified the difference in glutamate and aspartate concentration as significantly different with $\mathrm{p}<0.05$ between the cells grown with vehicle to UCPH-102 in all four cell lines. E) Identification of novel EAAT1-specific allosteric inhibitor. The left graph shows the effect of the inhibitor at $25 \mu \mathrm{M}$ on proliferation of the Kasumi-1 and T-ALL cell lines. The right histogram shows potency of drug at the indicated inhibitor concentrations on CCFR-CEM cells over the course of seven days. Each data point is relative to the starting number of cells and represents an average of three independent measurements \pm StDev. F) Model illustrating the function of mitochondrial EAAT1. 


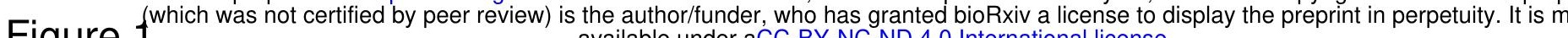
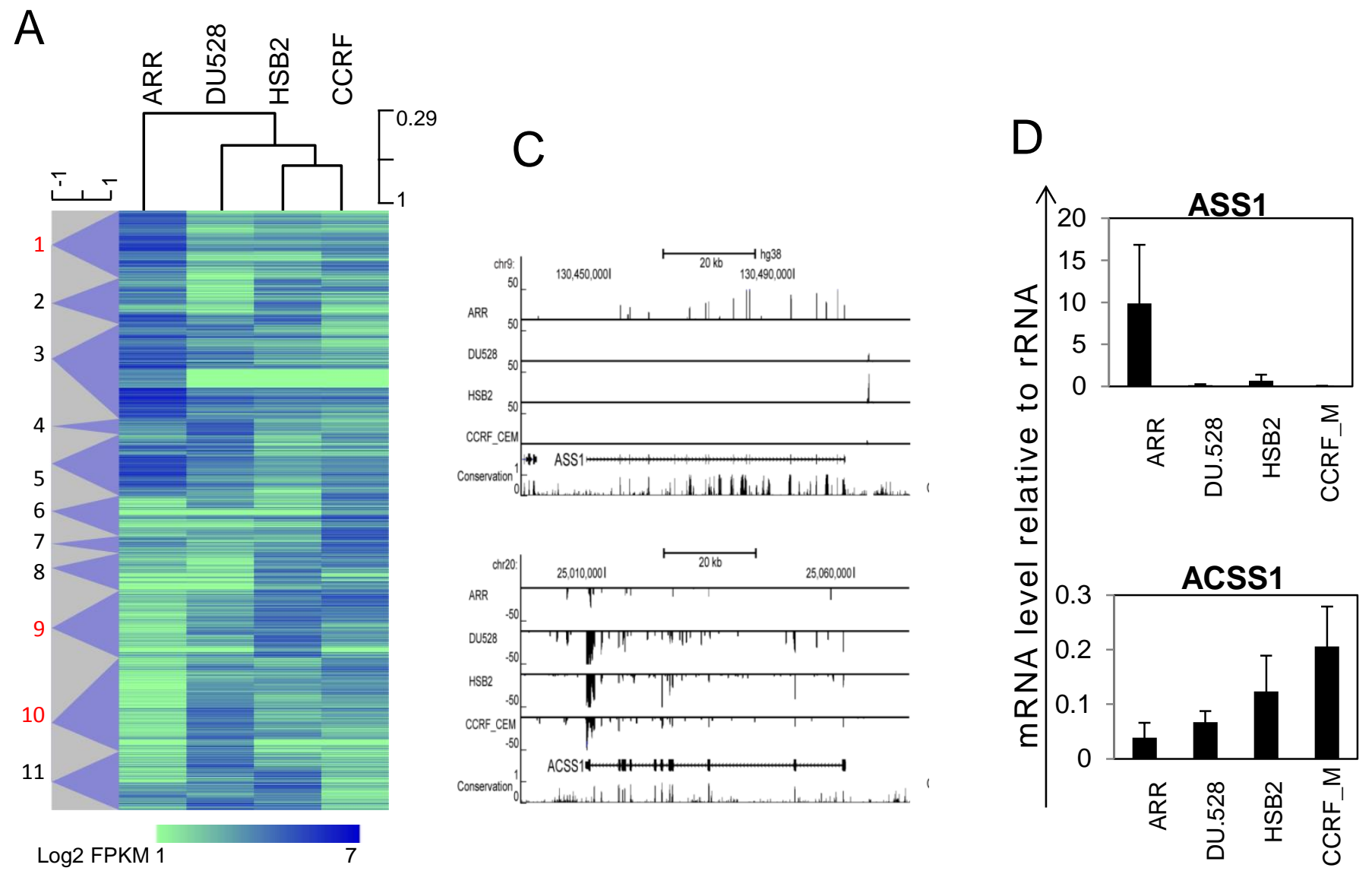

B

\section{Cluster_1_Genes Supressed in SIL-TAL}

$\%$ of genes

Biosynthesis of antibiotics

Thyroid hormone signaling pathway Protein processing in endoplasmic reticulum

Carbohydrate digestion and absorption Carbon metabolism Citrate cycle (TCA cycle) Aldosterone-regulated sodium reabsorption Metabolic pathways Biosynthesis of amino acids HIF-1 signaling pathway Aldosterone synthesis and secretion

Calcium signaling pathway

Central carbon metabolism in cancer Cholinergic synapse Ubiquitin mediated proteolysis Glutamatergic synapse Type II diabetes mellitus Pancreatic secretion
Cluster_9_Genes Upregulated in SIL-TAL \% of genes

T cell receptor signaling pathway Rap1 signaling pathway Neurotrophin signaling pathway Axon guidance Primary immunodeficiency

\section{0} 2 4

\section{Cluster 10 Genes Upregulated in SIL-TAL}

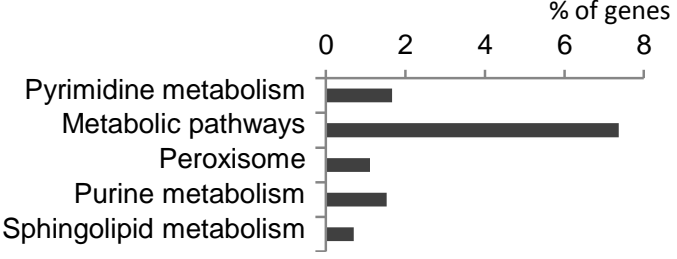




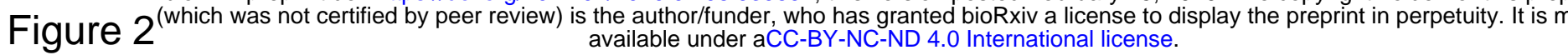

Figure $2^{\text {(which was not certified by peer review) is the author/funder, who has granted bioRxiv a license to display the preprint in perpetuity. It is } m}$
available under aCC-BY-NC-ND 4.0 International license.

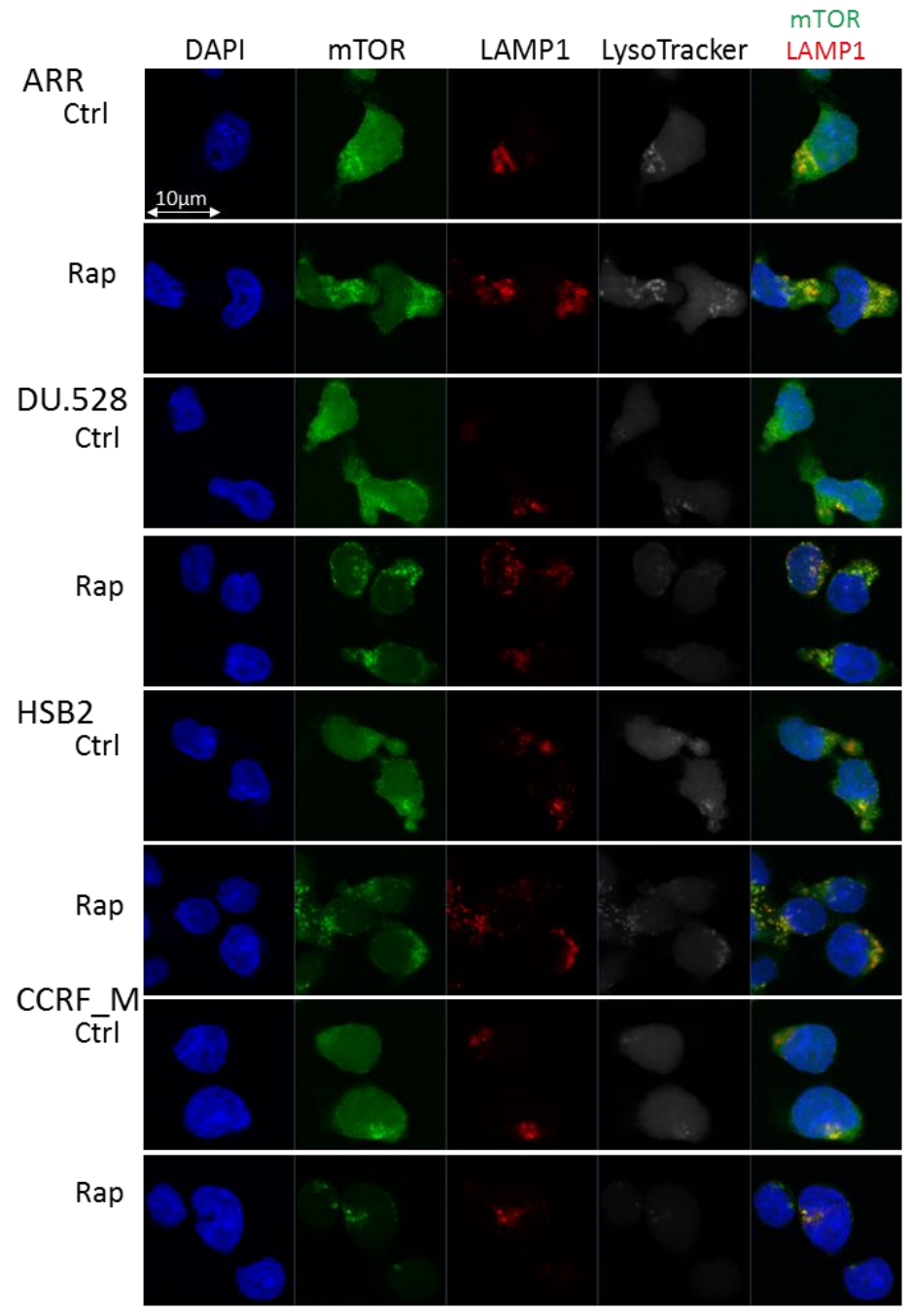

HSB2

CCRF_M

DU.528

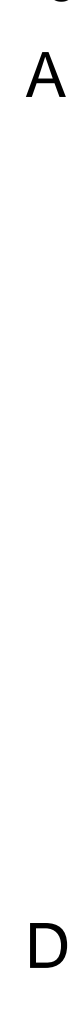

A
D
D

A
D
D

B

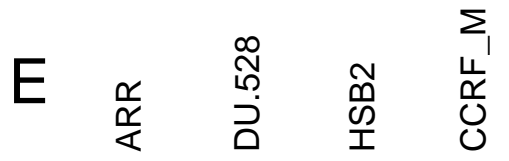

$-\quad+\quad+\quad+\quad-\quad+$ rapamycin

-HE⿵冂卄-Raptor5792

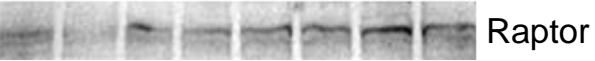

$\longrightarrow-\infty-\beta$-actin

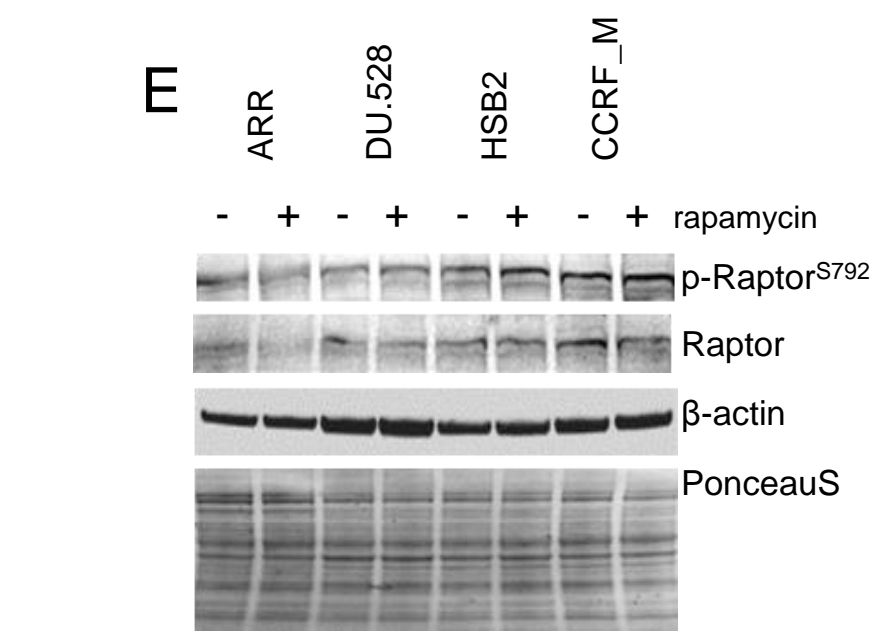

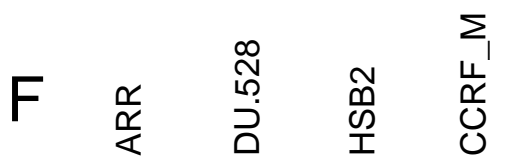

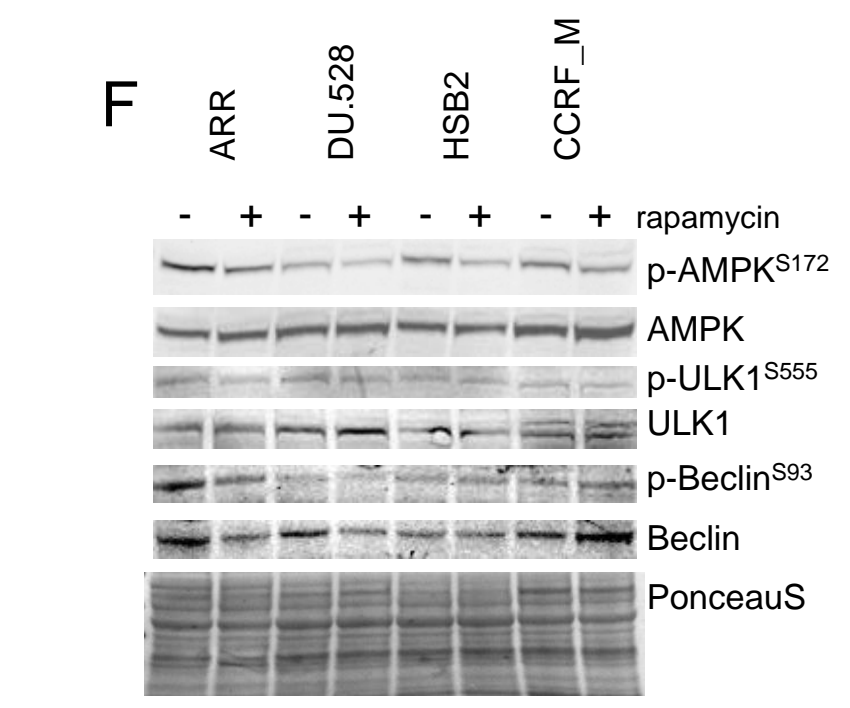

C
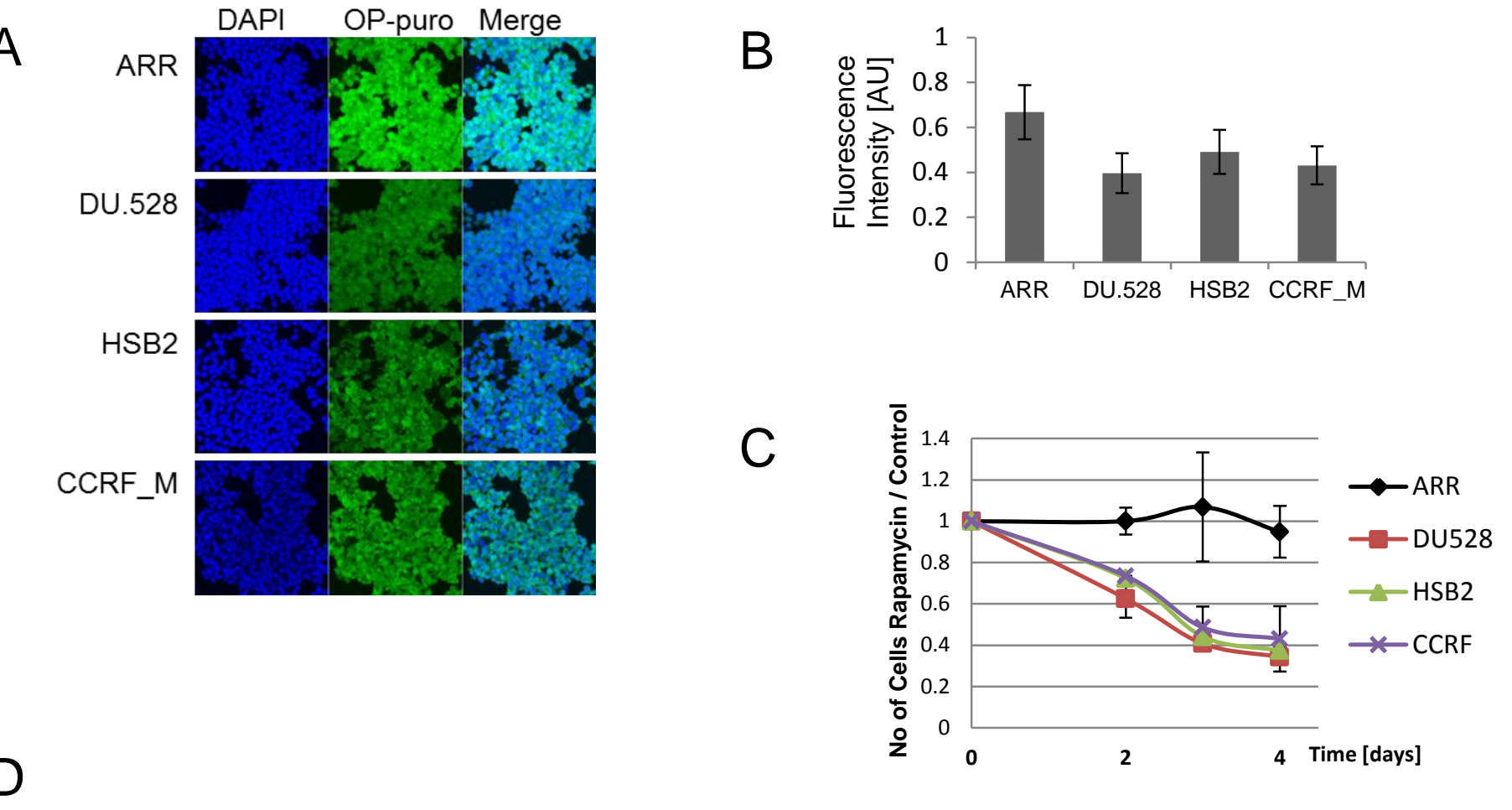

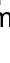



Figure $3^{\text {(which was not certified by peer review) is the author/funder, who has granted bioRxiv a license to display the preprint in perpetuity. It is } \mathrm{m}}$

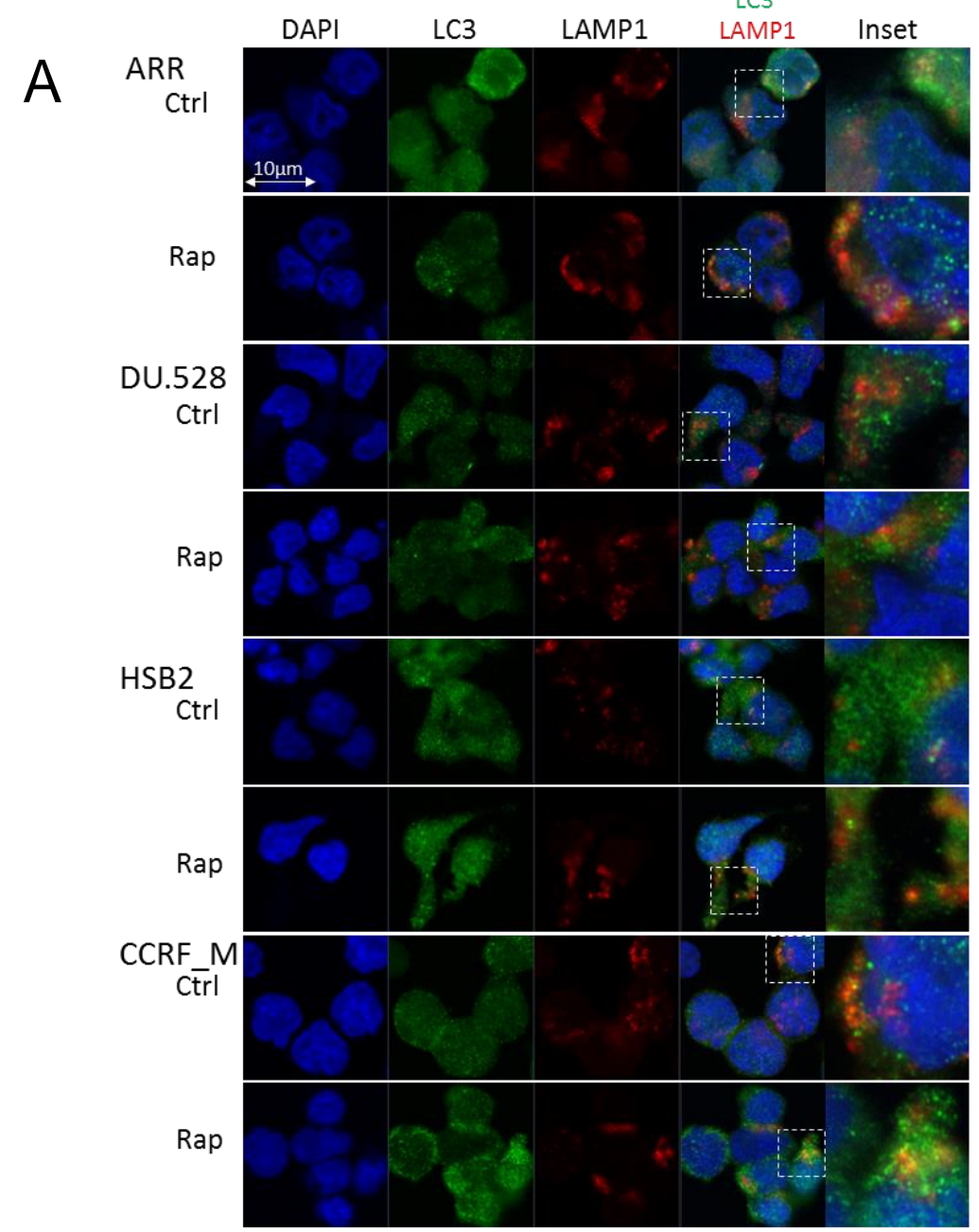

C

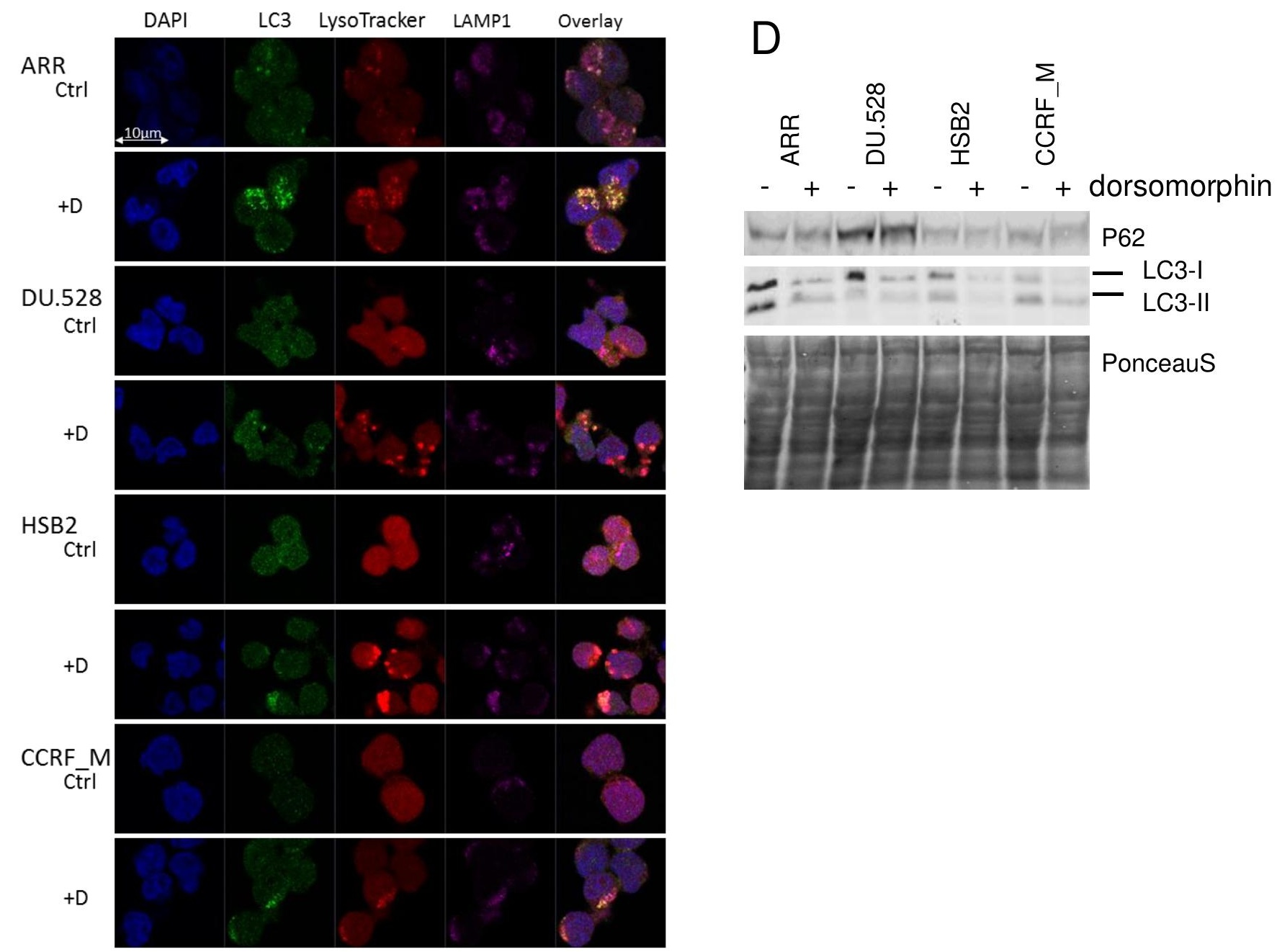




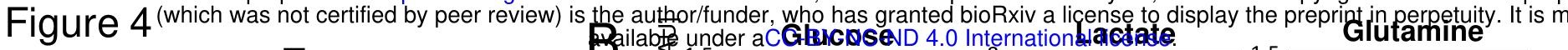
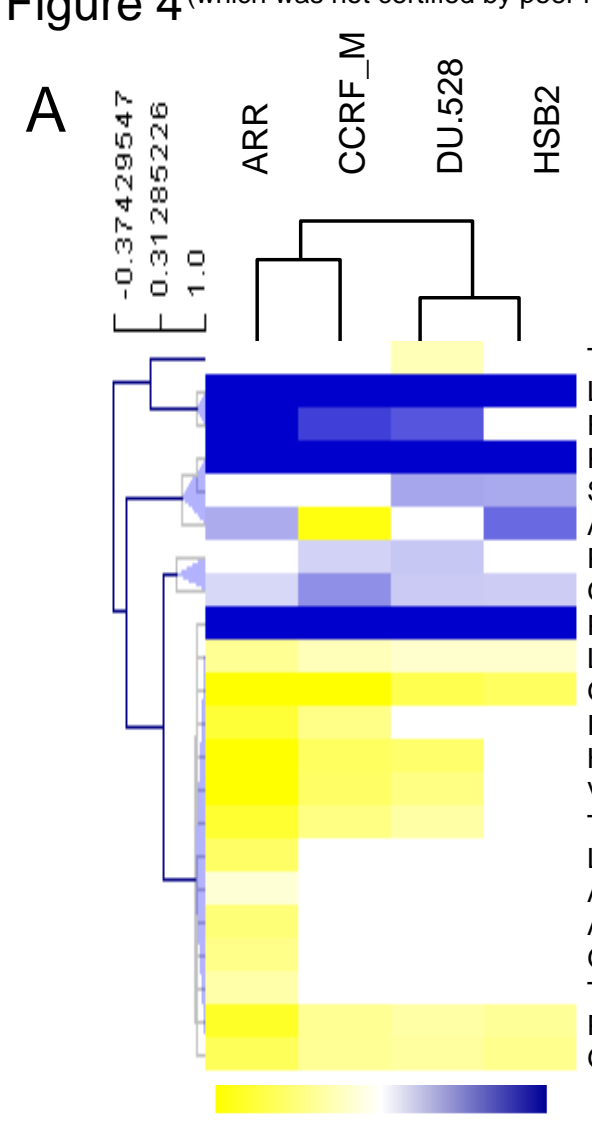

$\begin{array}{lll}-1 & 0 & 1\end{array}$

Log2 Medium+Cells / Medium
Threonine

Lactate

Formate

Pyruvate

Serine

Alanine

Proline

Glutamate

Pyroglutamate

Lysine

Glucose

Isoleucine

Histidine

Valine

Tyrosine

Leucine

Arginine

Aspartate

Glycine

Tryptophan

Phenylalanine

Glutamine
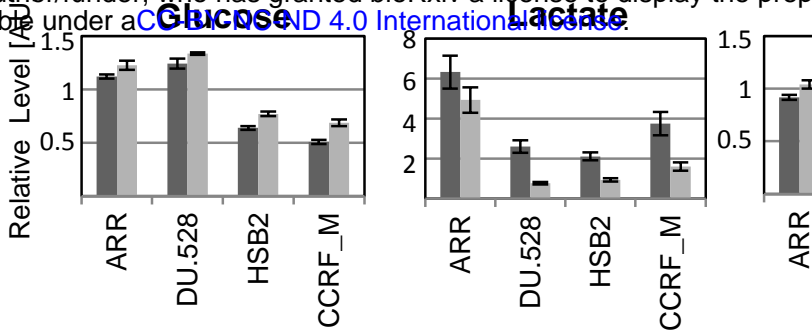

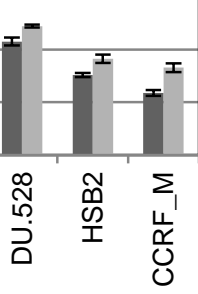

0

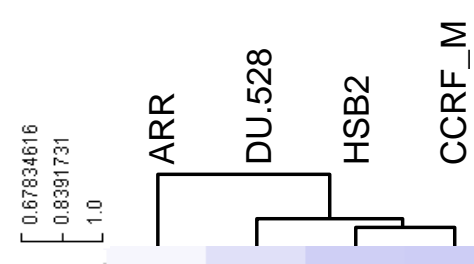

1

3

4

MC: Alanine

1

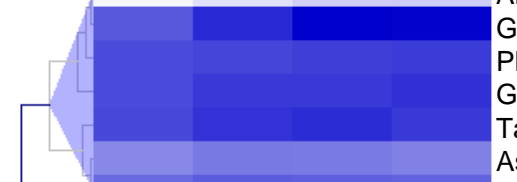

GPC

Phosphatidylcholine Glutamate

Taurine

Aspartate

ATP/ADP

Myoinositol

Fumarate

UDP-GaINAC

UDP-GICNAC

UDP-Glucose

Glutamine

Creatine

Phosphocreatine

Succinate

AMP

$\mathrm{NAD}+$

Glutathione

Leucine

Glycine

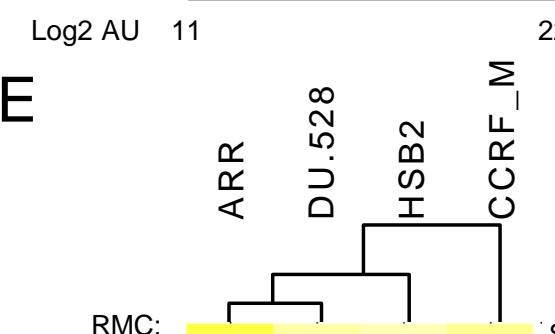

Isoleucine

Leucine

Phenylalanine

Valine

NAD+

Glutamine

Aspartate

UDP-GIcNAc

GPC

UDP-Galactose

UDP-Glucose

Alanine

UDP-GalNAc

Glutathione

Glutamate

Phosphocreatine

Fumarate

Taurine

ATP/ADP

Phosphatidylcholine

Lactate

Acetate

Glycine

AMP

Succinate

GXP

Creatine

Myoinositol
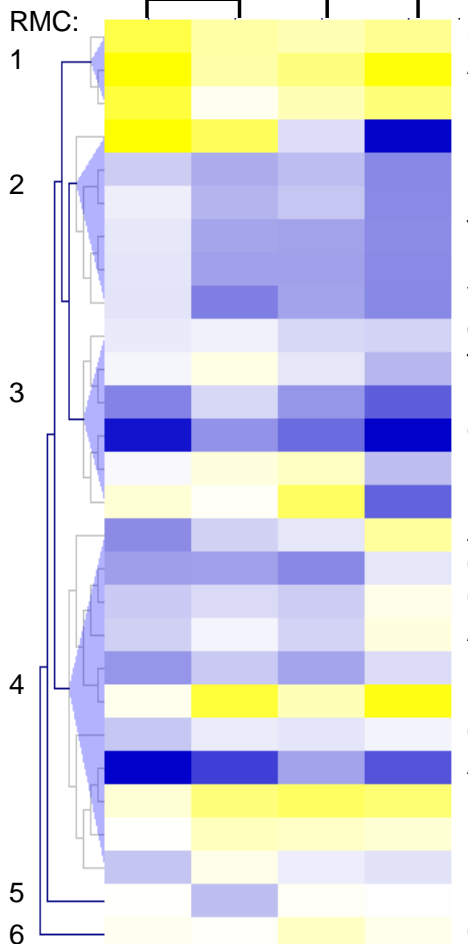

Succinate

AMP

UMP

Lactate

Phenylalanine

Leucine

Tyrosine

Isoleucine

Valine

Creatine

Taurine

Myoinositol

Glutamine

UDP-GIcNAc

UDP-GalNAc

Aspartate

Glycine

Glutamate

Acetate

Benzoate

Phosphatidylcholine

GPC

Alanine

UDP-Glucose

Fumarate

Phosphocreatine

NAD+

Glutathione 


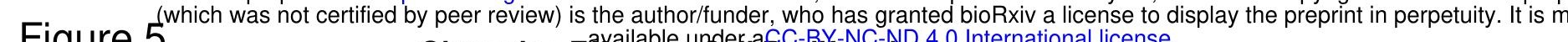

Figure 5

B
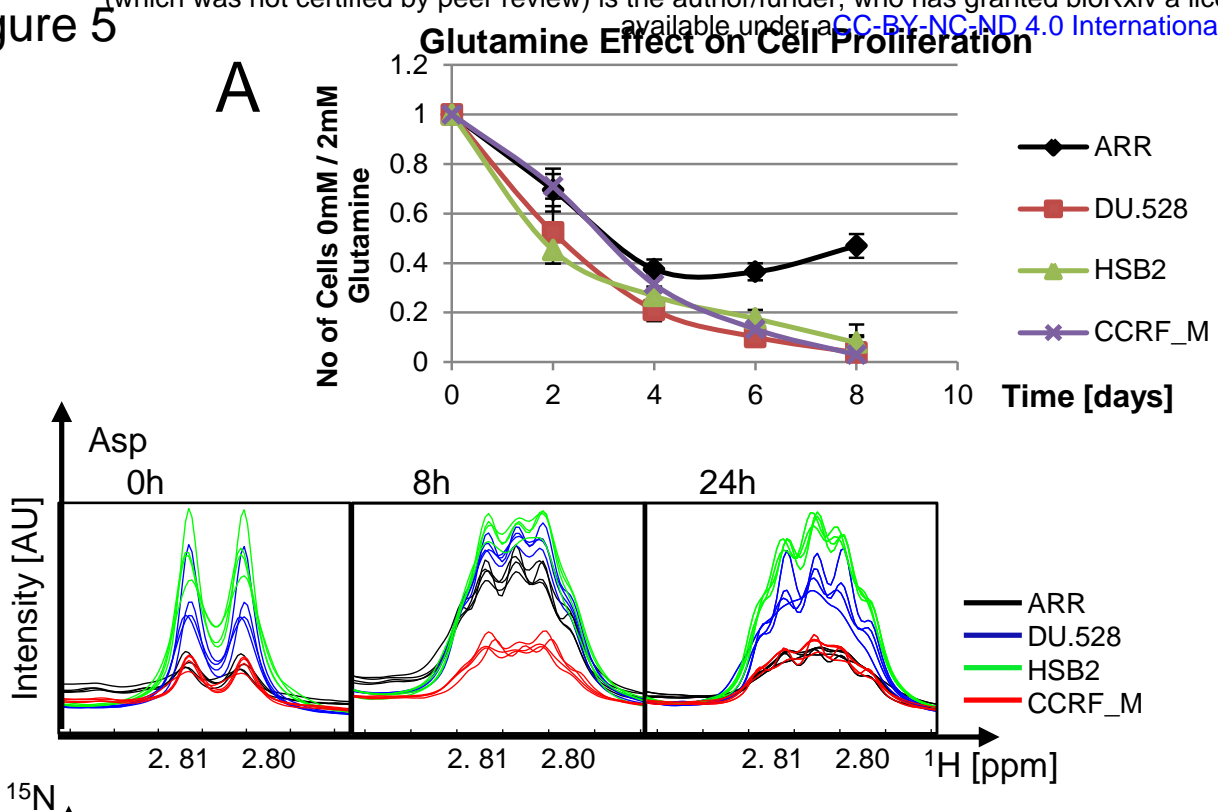

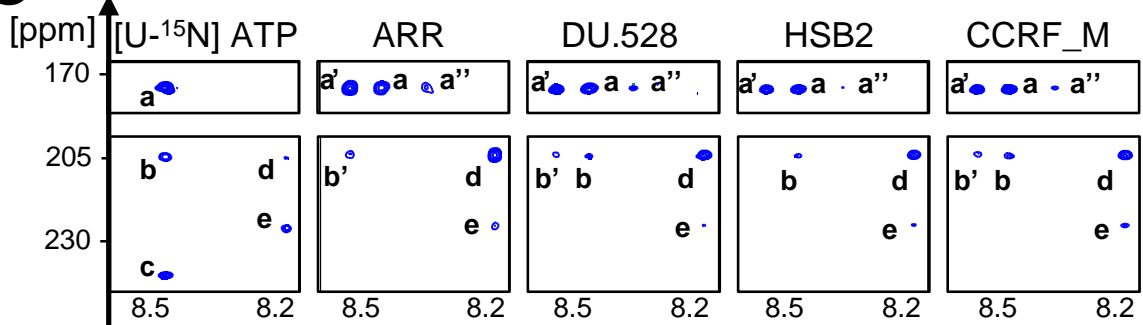
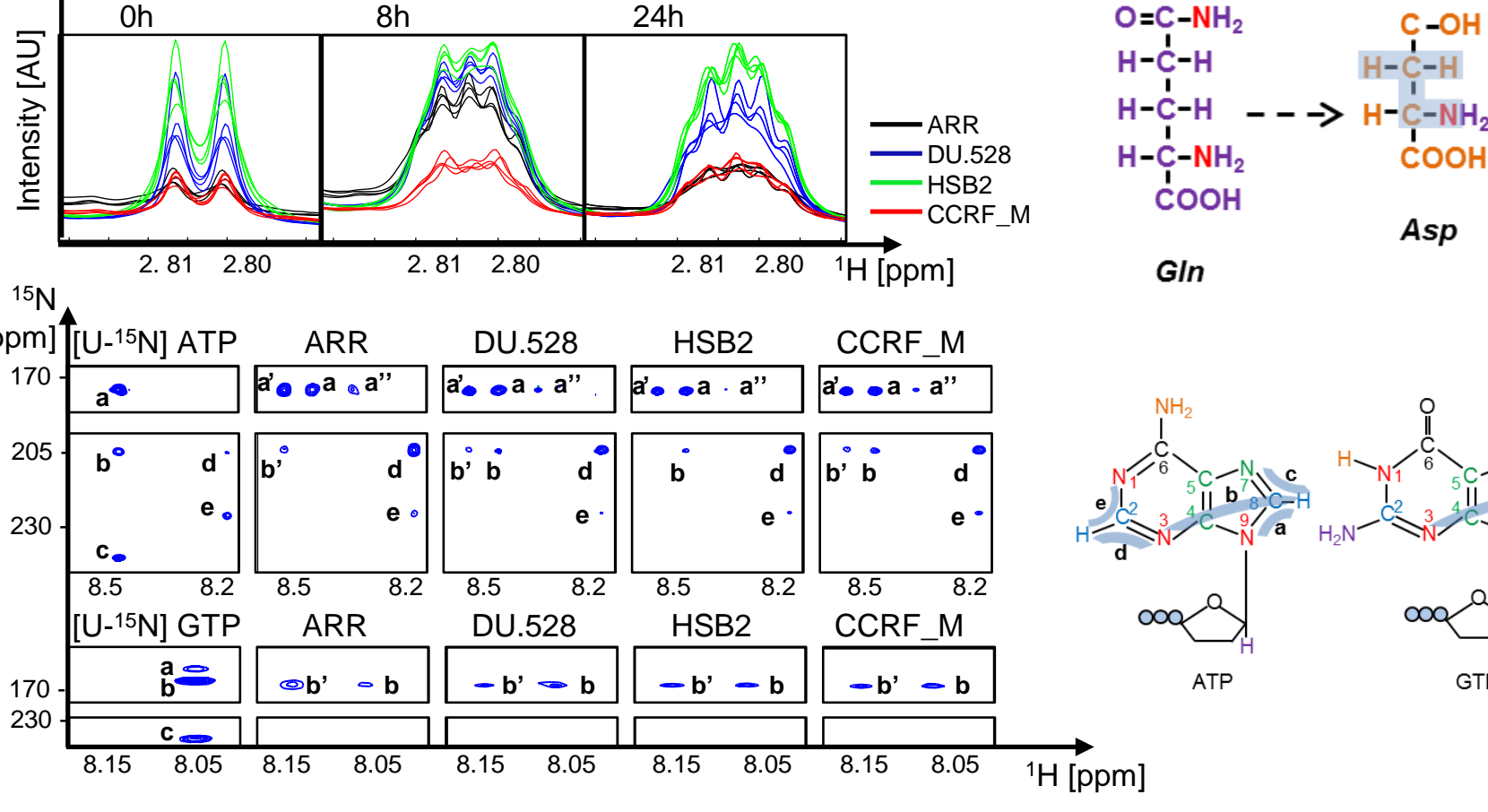

GIn

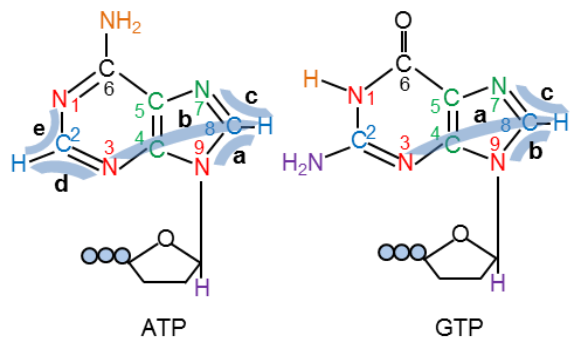

D
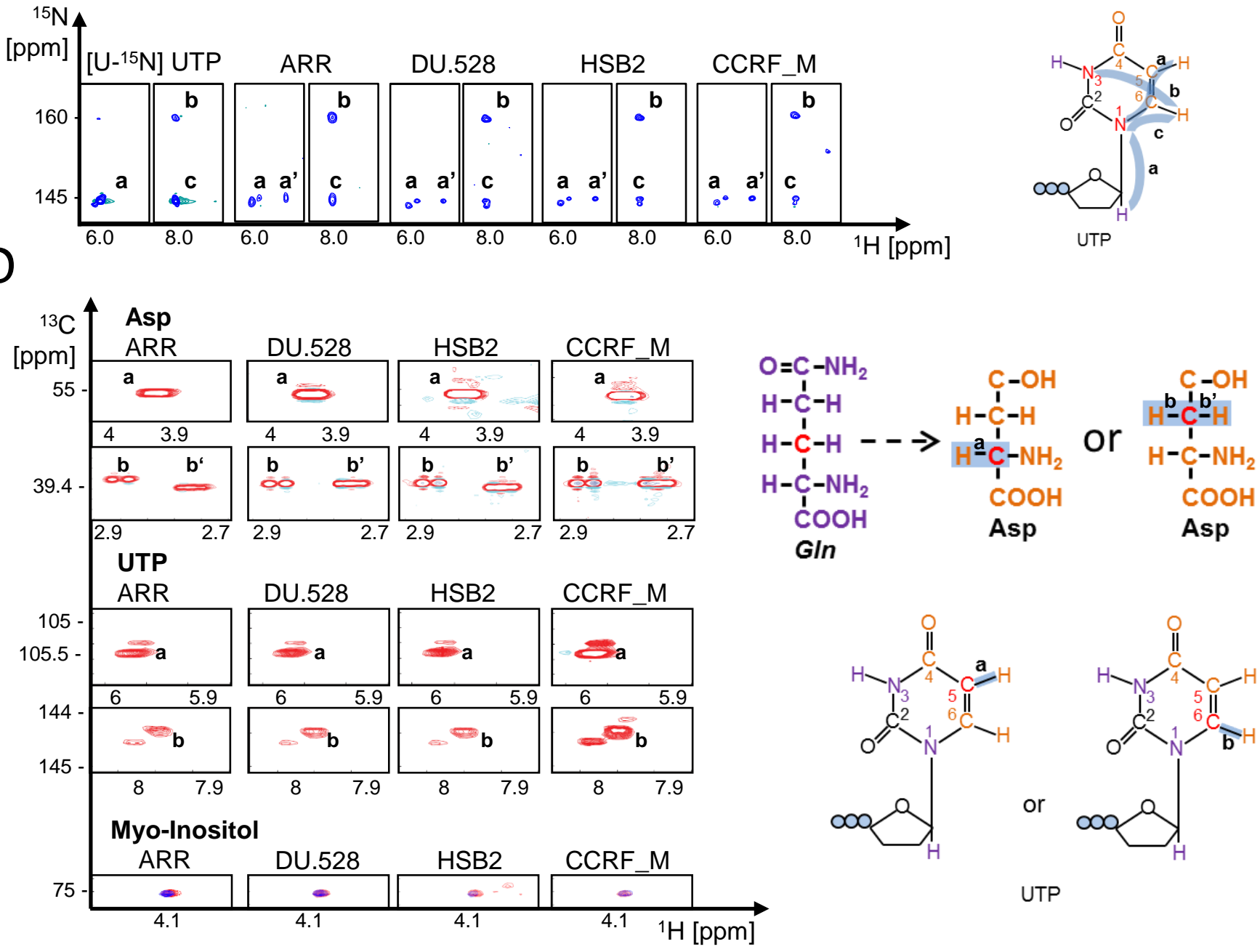
$\mathrm{F}$

(which was not certified by peer review) is the author/funder, who has granted bioRxiv a license to display the preprint in perpetuity. It is

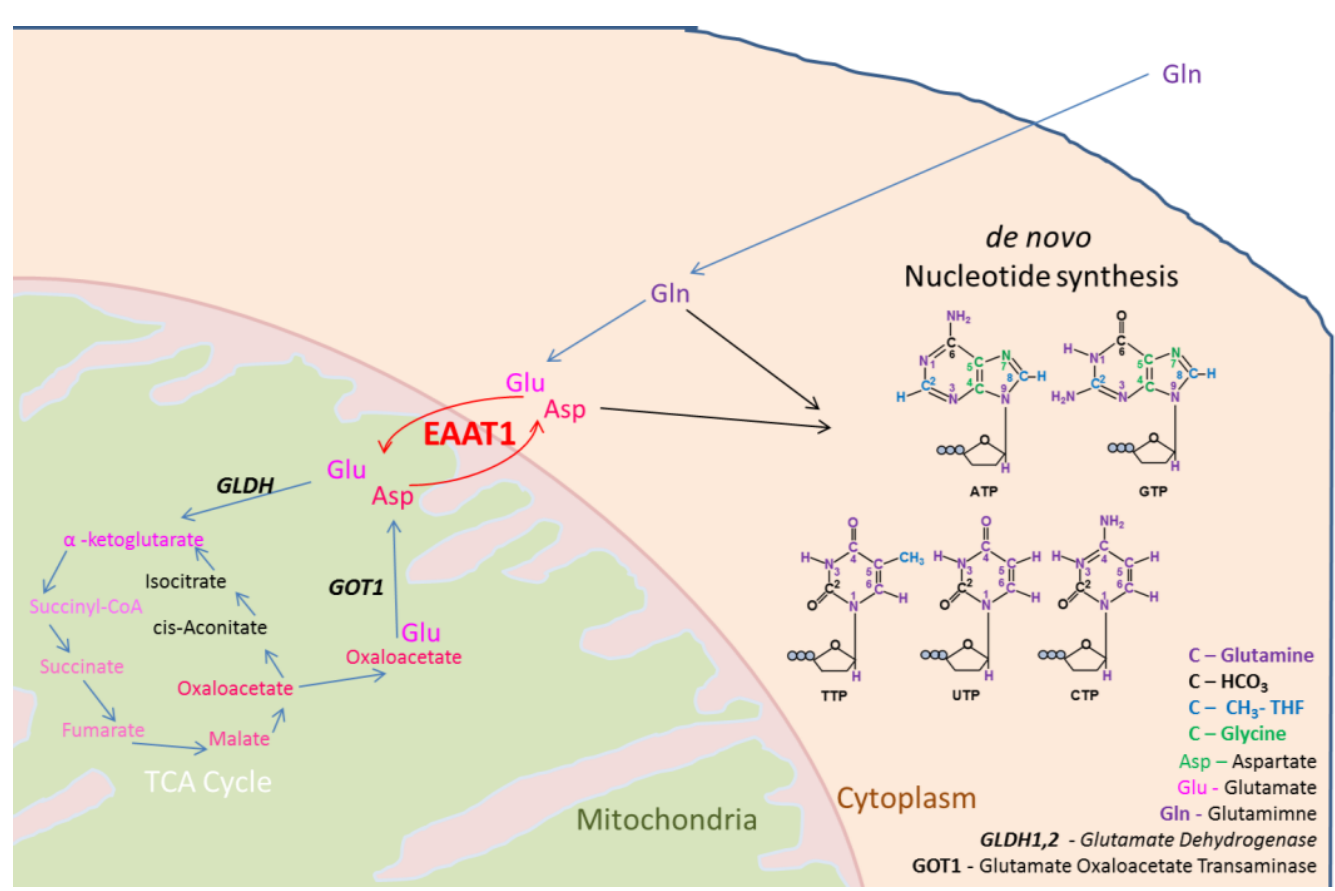


A

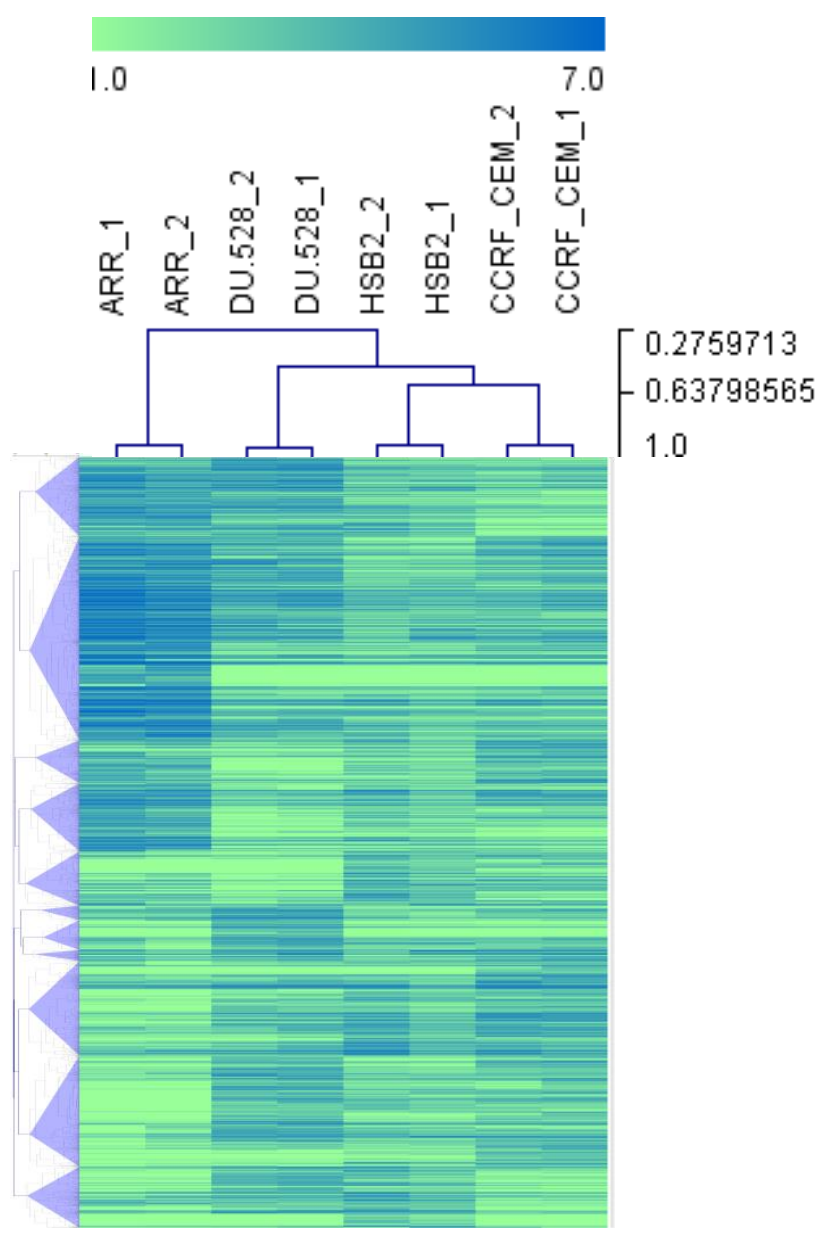

B

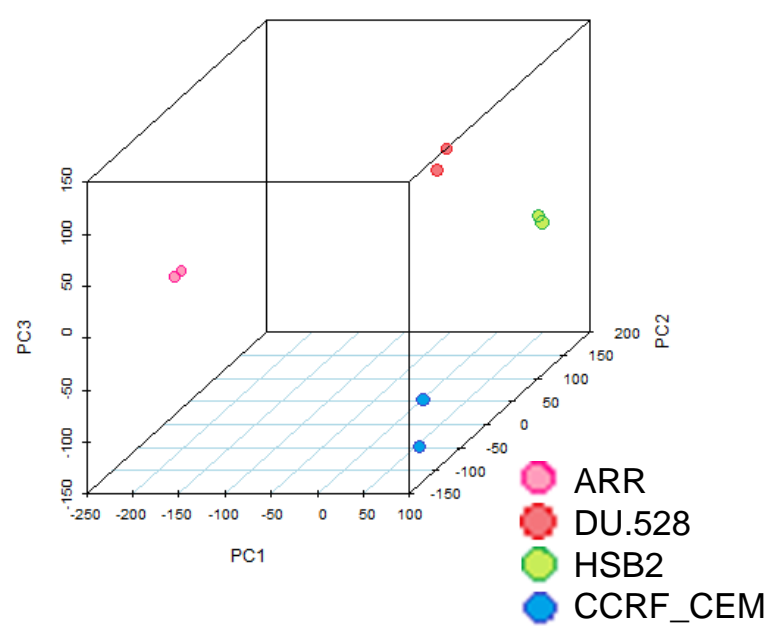


Cluster_8_Genes Upregulated in HSB2 and CCRF_M

\section{Cluster_11_Genes Upregulated in DU.528 and HSB2}

T cell receptor signaling pathway MAPK signaling pathway Central carbon metabolism in cancer Hippo signaling pathway Endometrial cancer Signaling in stem cells pluripotency

Rap1 signaling pathway

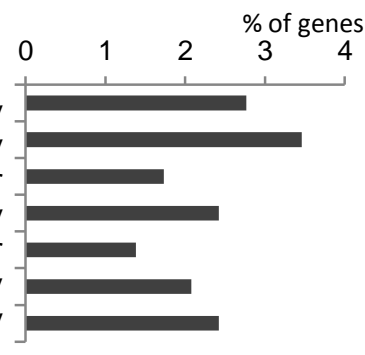

Natural killer cell mediated cytotoxicity Acute myeloid leukemia Pathways in cancer TNF signaling pathway MAPK signaling pathway Transcriptional misregulation in cancer

$T$ cell receptor signaling pathway

Type II diabetes mellitus Osteoclast differentiation Fc epsilon RI signaling pathway

Prolactin signaling pathway Insulin signaling pathway

Neurotrophin signaling pathway Small cell lung cancer Insulin resistance

Central carbon metabolism in cancer

FoxO signaling pathway Renal cell carcinoma

NF-kappa B signaling pathway $B$ cell receptor signaling pathway

Ras signaling pathway Jak-STAT signaling pathway Chronic myeloid leukemia AMPK signaling pathway Focal adhesion

Rap1 signaling pathway

Regulation of actin cytoskeleton Toll-like receptor signaling pathway Hepatitis C Colorectal cancer Progesterone-mediated oocyte maturation Proteoglycans in cancer Glioma Pancreatic cancer Influenza A

Viral carcinogenesis Melanoma

Chemokine signaling pathway Choline metabolism in cancer

Platelet activation

Endometrial cancer Non-small cell lung cancer Signaling in stem cells pluripotency Fc gamma R-mediated phagocytosis mTOR signaling pathway Hepatitis B 

available under aCC-BY-NC-ND 4.0 International license.

A

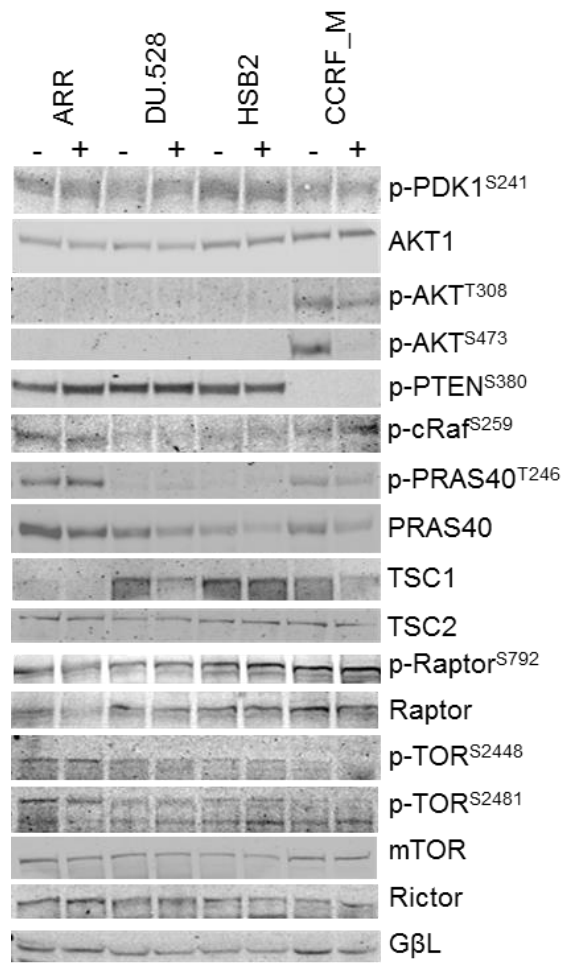

C

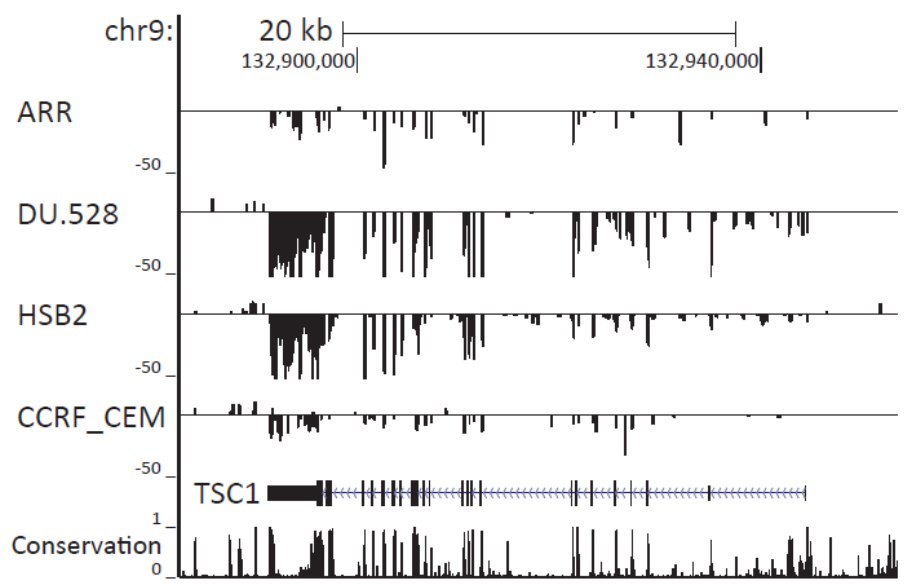

D

\begin{tabular}{ccccccc} 
& \multicolumn{1}{c}{ TSC1 } & \multicolumn{4}{c}{ FPKM } \\
\multicolumn{1}{c}{} & tss_id & ARR & DU528 & HSB2 & CCRF_CEM \\
gene & TSS126025,-26,-27 & 5.15 & 13.83 & 11.73 & 5.57 \\
transcript & TSS126025 & 0.00 & 6.11 & 0.01 & 2.38 \\
transcript & TSS126025 & 0.94 & 3.50 & 1.47 & 0.00
\end{tabular}

E

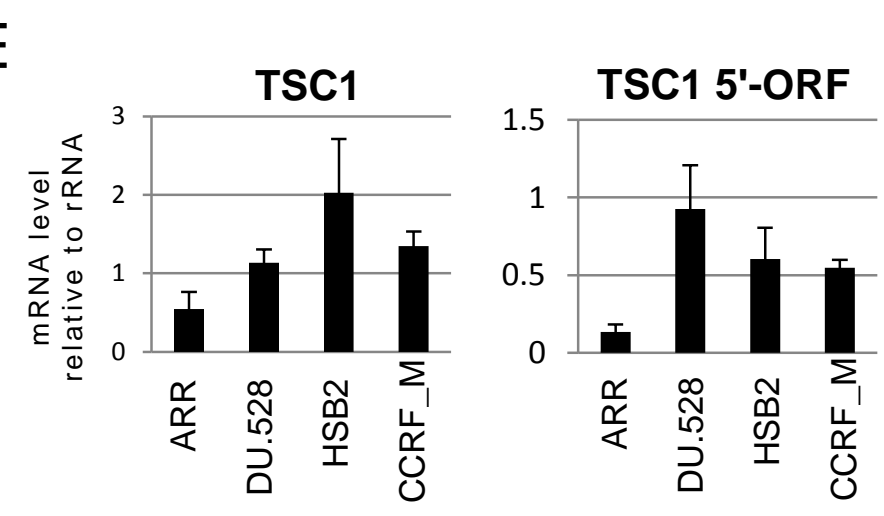

B

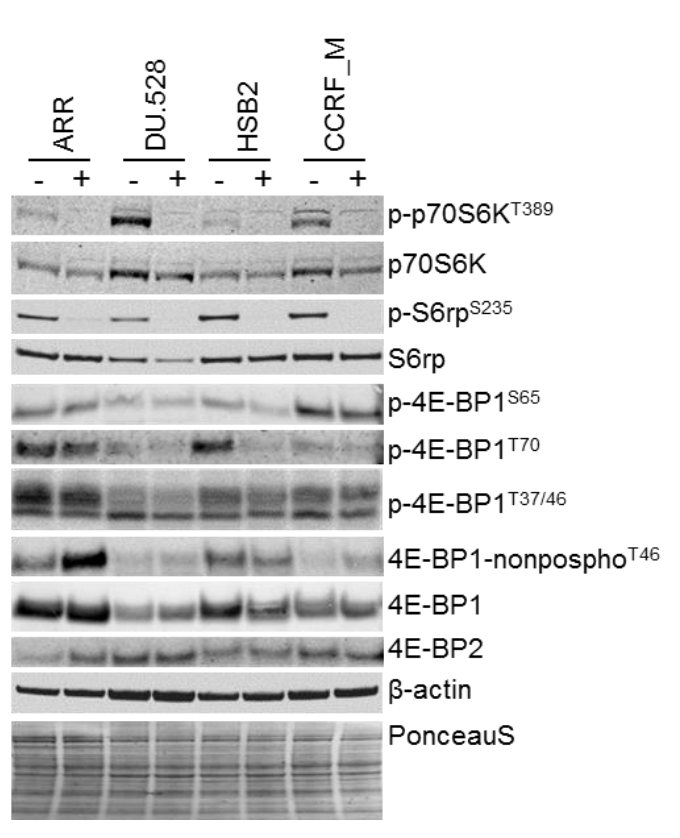

(which was not certified by peer review) is the author/funder, who has granted bioRxiv a license to display the preprint in perpetuity. It is $m$ 

Fiaure (which was not certified by peer review) is the author/funder, who has granted bioRxiv a license to display the preprint in perpetuity. It is $\mathrm{m}_{\text {available under aCC-BY-NC-ND } 4.0 \text { International license. }}$

Figure S4
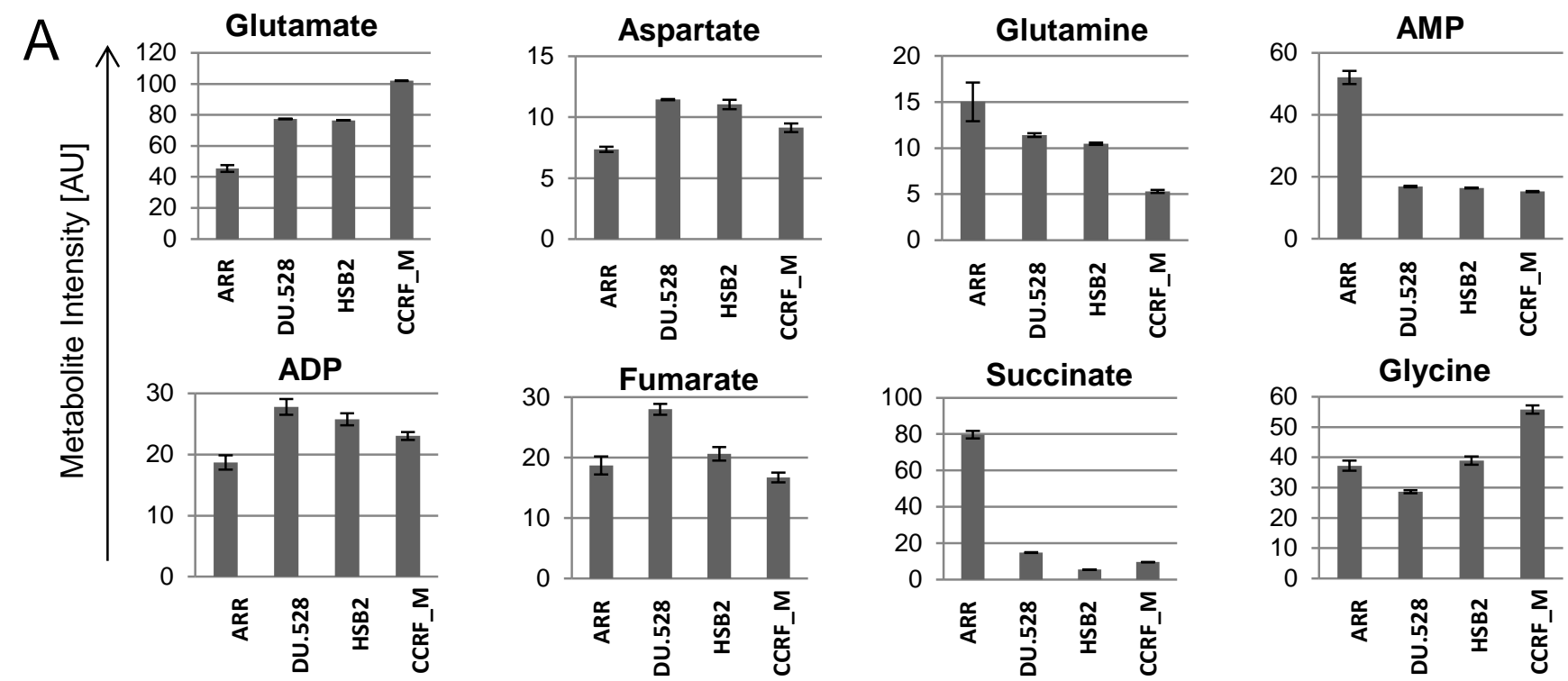

B
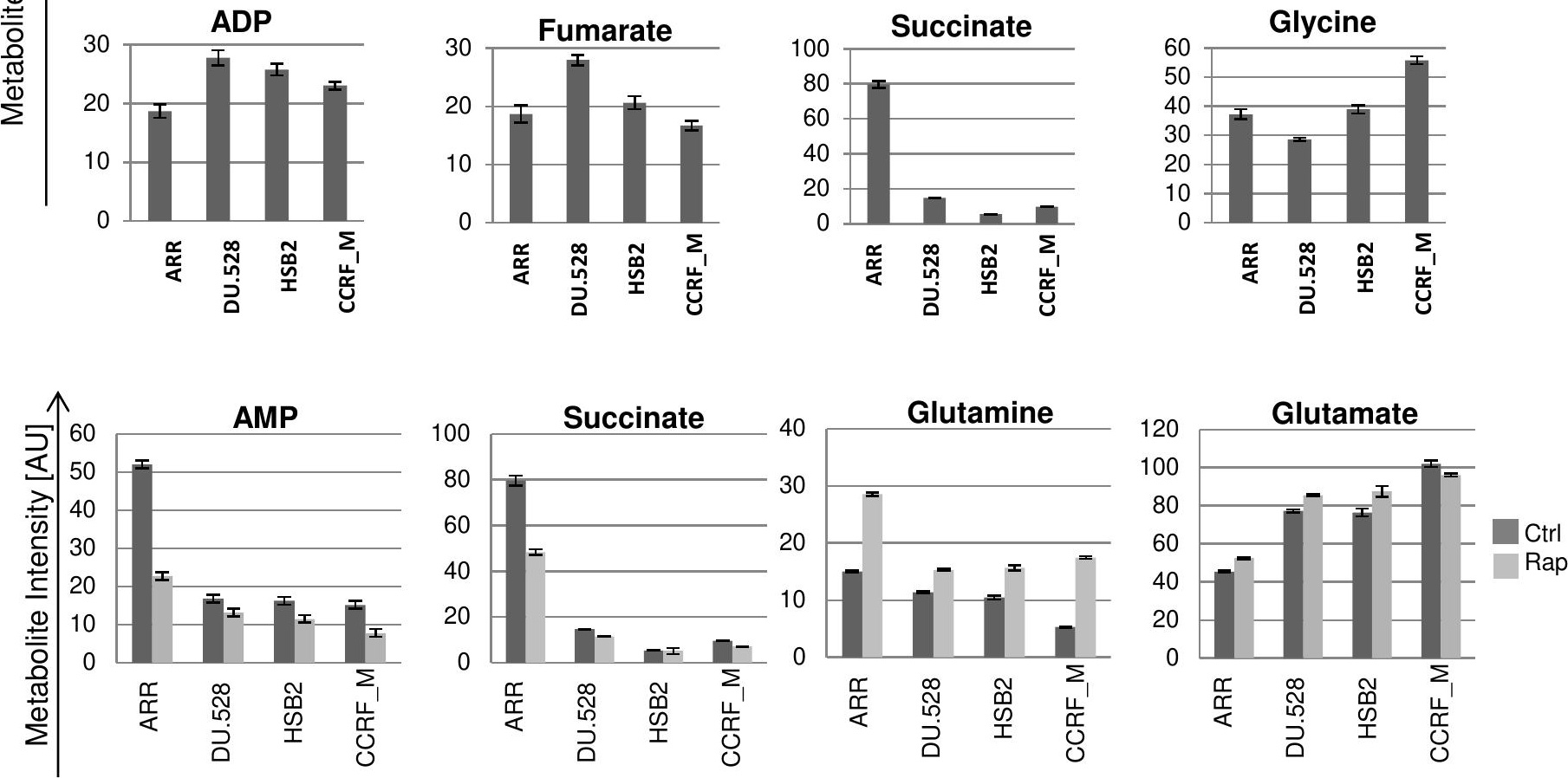


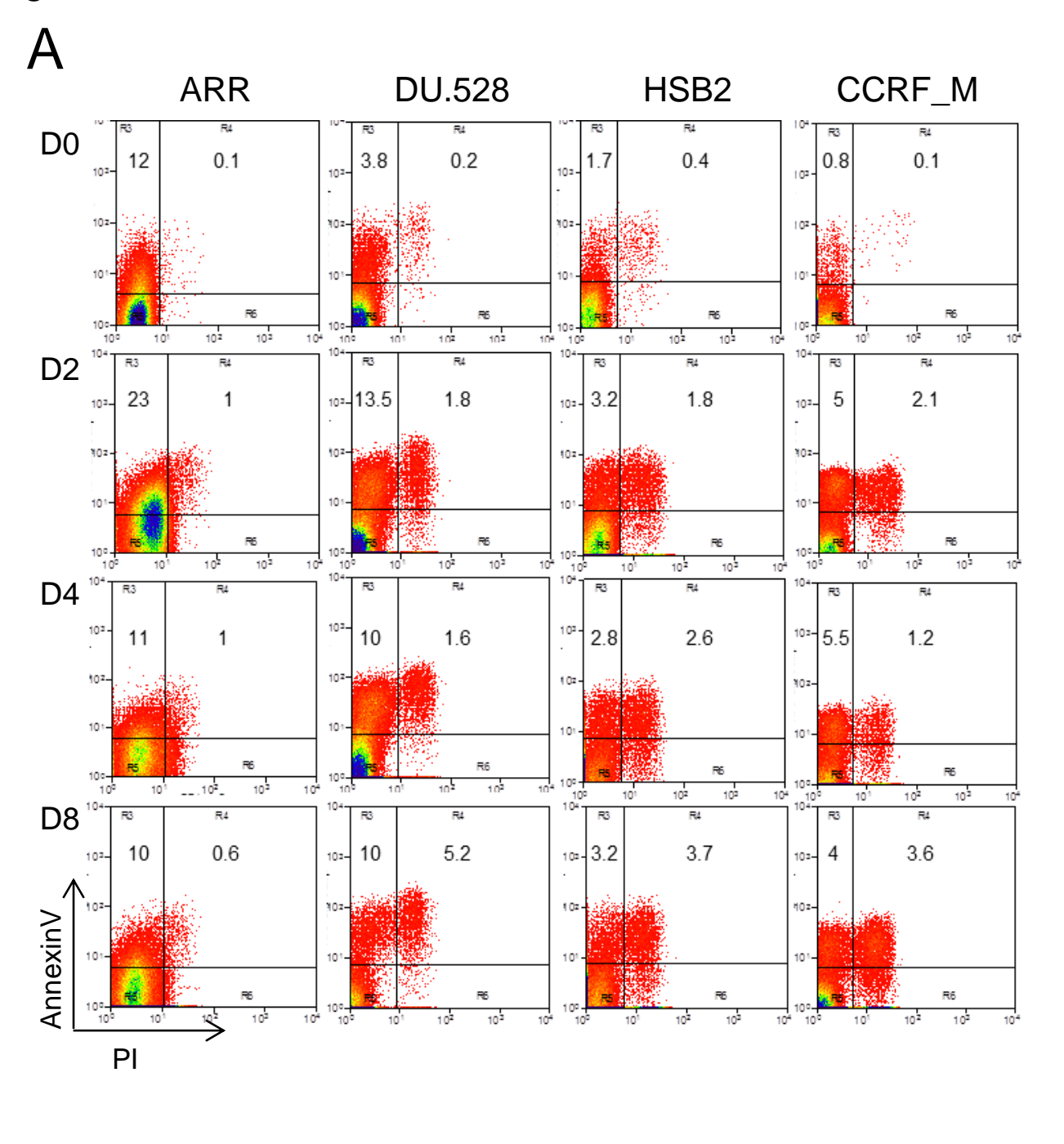

Figure $\$ 55$ which was not certified by peer review) is the author/funder, who has granted bioRxiv a license to display the preprint in perpetuity. It is
available under aCC-BY-NC-ND 4.0 International license.
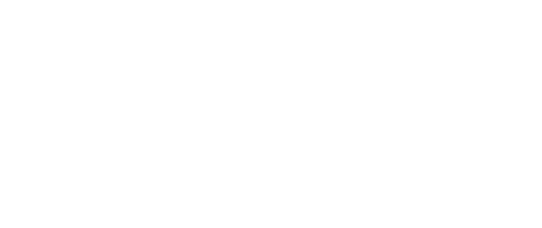

PI 
Figure $S^{(2 h}$

A available under aCC-BY-NC-ND 4.0 International license.
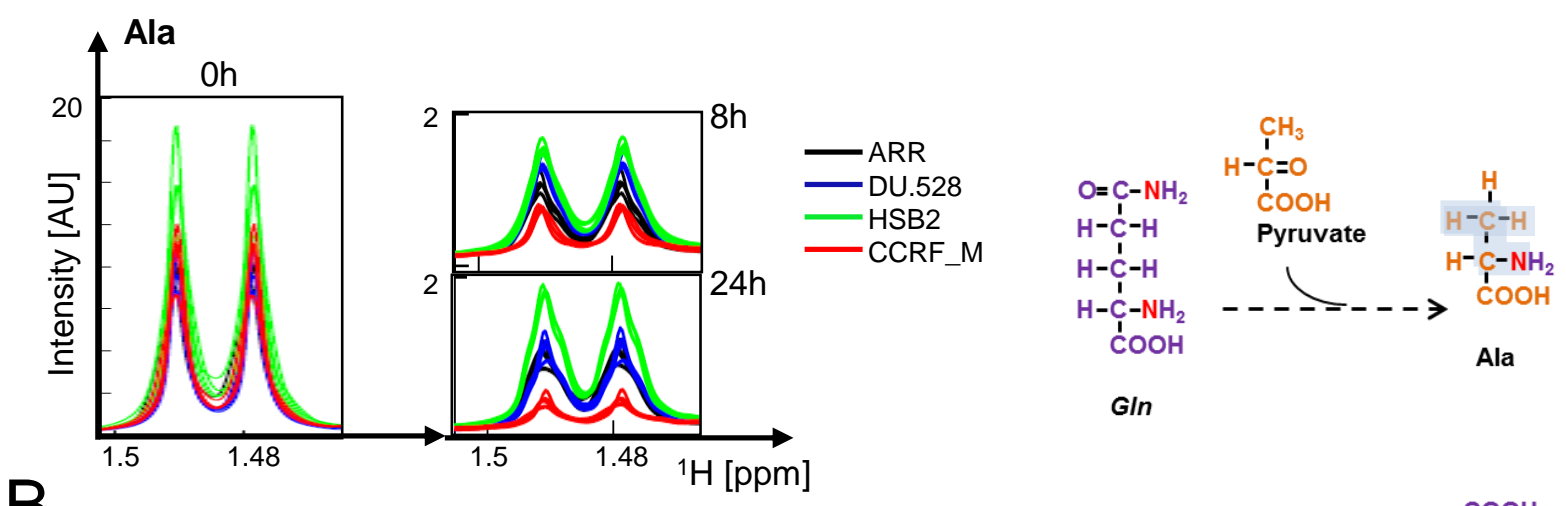

\section{B}

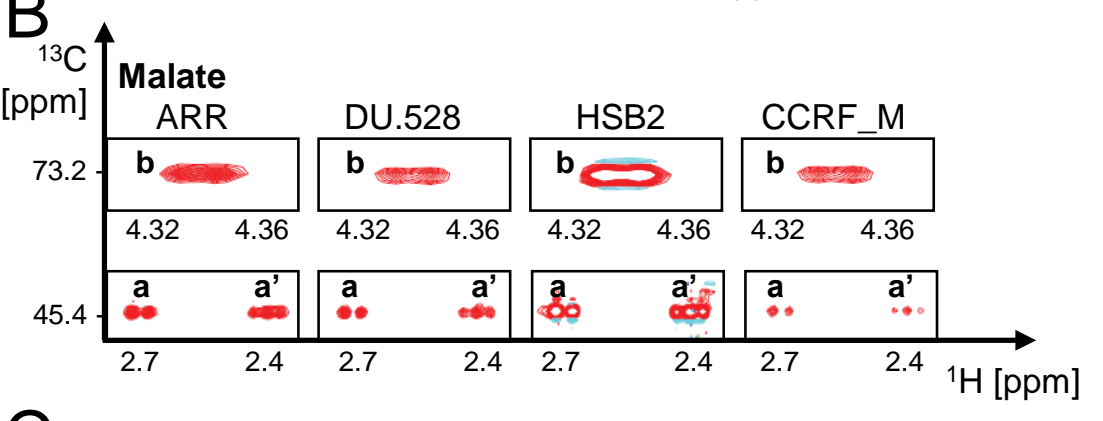

C
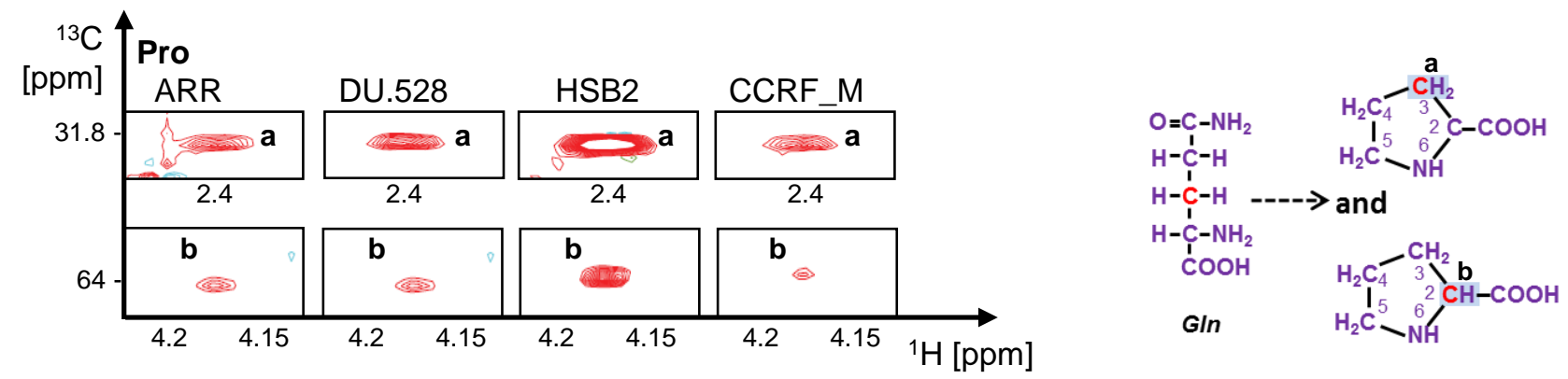

D
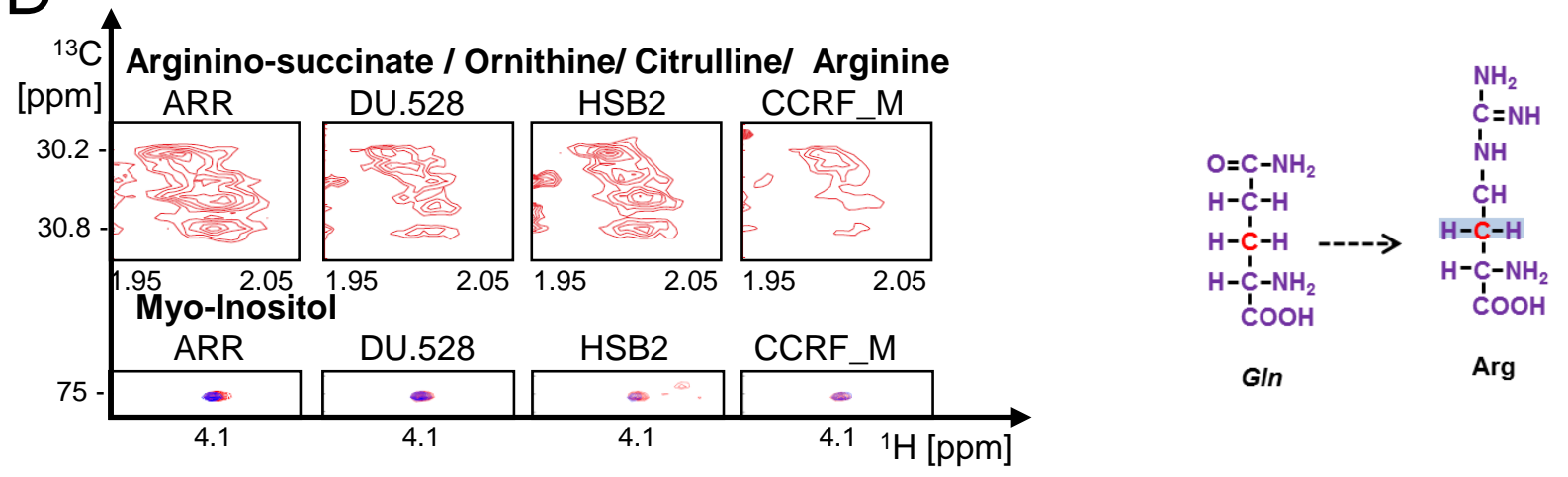

E
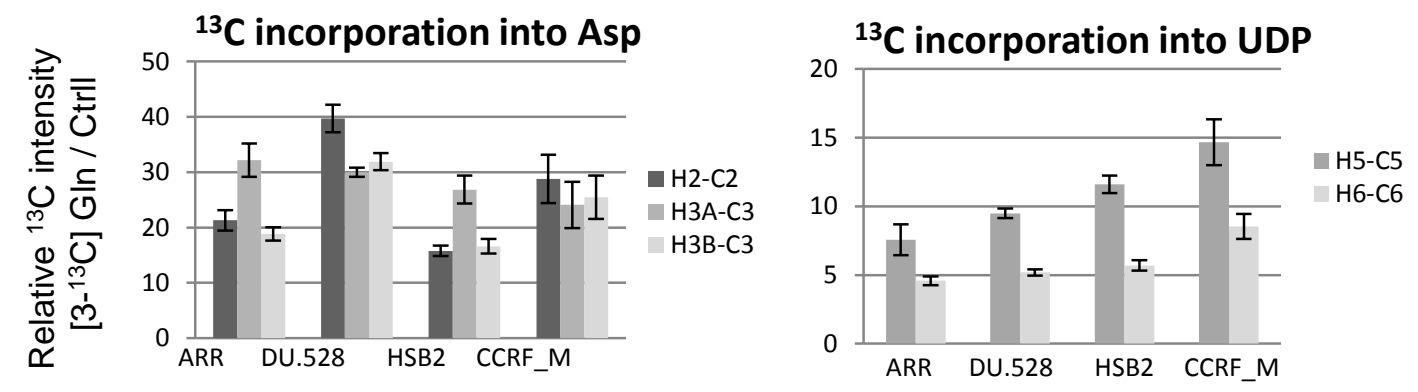
